\title{
Effects of Size, Composition, and Evolutionary Pressure in Heterogeneous Cournot Oligopolies with Best Response Decisional Mechanisms
}

\author{
Fausto Cavalli, ${ }^{1}$ Ahmad Naimzada, ${ }^{1}$ and Marina Pireddu ${ }^{2}$ \\ ${ }^{1}$ Department of Economics, Management and Statistics, University of Milano-Bicocca, \\ 20126 Milano, Italy \\ ${ }^{2}$ Department of Mathematics and Applications, University of Milano-Bicocca, 20125 Milano, Italy
}

Correspondence should be addressed to Marina Pireddu; marina.pireddu@unimib.it

Received 12 November 2014; Revised 31 March 2015; Accepted 31 March 2015

Academic Editor: Baodong Zheng

Copyright (C) 2015 Fausto Cavalli et al. This is an open access article distributed under the Creative Commons Attribution License, which permits unrestricted use, distribution, and reproduction in any medium, provided the original work is properly cited.

\begin{abstract}
We study heterogeneous Cournot oligopolies of variable sizes and compositions, in which the firms have different degrees of rationality, being either rational firms with perfect foresight or naive best response firms with static expectations. Each oligopoly can be described using its size and composition, that is, the fraction of firms that are rational. We take into account two frameworks, one in which the decisional rules are exogenously assigned and the other in which the firms may change their heuristics. We consider a switching mechanism based on a logit rule, where the switching propensity is regulated by a parameter which represents the evolutionary pressure. In the fixed fractions setting, we prove that, in general, the composition has a stabilizing effect, while increasing the oligopoly size leads to instability. However, we show that, for particular parameters settings, stability is not affected by the composition or the firms number. Similarly, in the evolutionary fractions setting, we analytically prove that when marginal costs are identical, increasing the evolutionary pressure has a destabilizing effect. Nevertheless, focusing on particular examples with different marginal costs we are able to show that evolutionary pressure may also have a stabilizing or a neutral role.
\end{abstract}

\section{Introduction}

An oligopoly is a market structure which consists of only a few firms that, in order to decide their productions, take into account at the same time both their own actions and those of their competitors. Since the first formal theory of oligopoly developed by Cournot in 1838 (see [1]), the research focused on the decisional mechanisms that firms may adopt in choosing their strategies and on the different behaviors of the oligopoly models obtained combining such rules, in particular when the firms are not fully rational, so that they have to adjust their production level over time. In this case, the oligopolistic competition can be modeled using a discrete dynamical system. The investigation about oligopolistic dynamical models can be subdivided into two research strands, concerning homogeneous oligopolies, in which all the firms adopt the same adjustment mechanism, and heterogeneous oligopolies, in which there are at least two firms that adopt different decisional mechanisms.
Starting from the seminal contributions by Palander [2] and Theocharis [3], several homogeneous frameworks have been investigated (see, e.g., the works by Lampart [4], Matsumoto and Szidarovszky [5], and Naimzada and Tramontana [6]), involving different economic contexts or behavioral rules. See [7] for a survey on nonlinear oligopolies and the references therein. The common feature of such models is that, from the equilibrium stability point of view, the situation can severely change when the oligopoly size increases. In particular, the size has in general a destabilizing role.

The literature about heterogeneous oligopolies, usually dealing with a limited and fixed in advance number of firms, has instead developed in the direction of studying the possible consequences arising from considering different rationality degrees, also on the stability of Nash equilibria. In particular, such works, in view of giving a foundation to the concept of Nash equilibrium, investigate under which conditions the dynamics converge towards the Nash equilibrium or towards 
a different attractor. To that research strand belong, for instance, the contributions by Agiza and Elsadany $[8,9]$, by Agiza et al. [10, 11], by Ahmed and Agiza [12], by Angelini et al. [13], by Bischi and Naimzada [14], by Canovas et al. [15], by Cavalli and Naimzada [16, 17], by Cavalli et al. [18, 19], by Elabbasy et al. [20], by Ji [21], by Li and Ma [22], by Matsumoto [23], by Naimzada and Sbragia [24], by $\mathrm{Pu}$ and $\mathrm{Ma}$ [25], by Tramontana [26], and by Tuinstra [27].

Our work aims to investigate the role of oligopoly size and composition for heterogeneous competitions. In particular, we consider an economy characterized by linear demand function and we assume linear total costs for the firms. The heterogeneity we study is in terms of the degree of rationality of the firms, as we consider two different kinds of informational endowments. Both the firms decide their production level using a best reply mechanism, in order to maximize their profits with respect to the production levels of all their competitors. However, rational firms have full informational and computational capabilities to solve such optimization problem and are endowed with perfect foresight, so that they exactly know their competitors next period strategies. On the other hand, naive best response firms are not able to predict such strategies, and thus they assume that their opponents next time production levels remain the same as in the current period, that is, they have static expectations. Finally, we allow each group of firms to have different marginal costs. The approach of considering the market split into two groups of firms, identical within each group, is common in the oligopoly literature, as shown, for instance, by the works of Anufriev et al. [28], Bischi et al. [29], Cavalli et al. [18], Droste et al. [30], and Matsumoto and Szidarovszky [5]. The theoretical rationale for such an approach is that although it concerns one of the simplest possible forms of heterogeneity among firms, besides keeping the study analytically tractable, it allows obtaining interesting results, for example, with respect to equilibrium stability and chaotic dynamics, which can be straightforwardly interpreted and linked to the heterogeneity in rationality.

In the adjustment mechanism of the less rational best response firms, because of capacity and financial constraints, we introduce a constraint on the strategy variation, so that firms can not immediately increase or decrease their production level by arbitrarily large quantities, but they adapt it towards best reply strategy with a certain reaction speed. To this end, we introduce a limiting mechanism similar to that used in [31] in microeconomic frameworks and in [32, 33] in macroeconomic settings. On the contrary, due to the high degree of rationality of the rational firms, we assume that they are able to freely modify their production level in every period.

We study two different settings, considering the case of fixed fractions, in which the behavioral rules are exogenously assigned and do not change, and that of evolutionary fractions, in which the firms can switch between the different mechanisms on the basis of the realized performances of the firms. To exploit the competition between all the oligopolists, we adopt a switching mechanism based on the logit rule, as proposed by Brock and Hommes in [34]. The performances of the behavioral rules are evaluated taking into account the profits that the rule allowed achieving in the previous period. In particular, we assign additional fixed informational costs to the rational firms, as a consequence of their high degree of rationality. The switching between mechanisms is regulated by the so-called evolutionary pressure parameter, which describes the propensity of a firm to switch to the most profitable rule.

In the existing literature, the study of heterogeneous oligopolies of generic size can be found in the works by Anufriev et al. [28], Banerjee and Weibull [35], Droste et al. [30], and Gale and Rosenthal [36]. However, only the setting and the aims of the contribution by Droste et al. are similar to those investigated in the present work, as in both papers the economy is characterized by the same linear demand function and the firms may be both rational and best response. However, in [30] it is considered an infinite population of firms that are randomly matched in pairs at each discrete-time period and that play a symmetric Cournot duopoly game, so that the influence of oligopoly size on the dynamics can not be studied and only the evolutionary fractions framework is investigated. Moreover, no production limiters are taken into account and a replicator switching mechanism is considered, which is different from that studied in our contribution. Another substantial difference is that in [30] firms compute their best response with respect to the average industry output, while in our work all the firms compete against each other. Finally, in [30] the firms have identical cost functions, while in our paper they may possibly be different.

About the fixed fractions model, our main results concern the effects of the oligopoly size and composition and of the evolutionary pressure on the stability of the equilibrium, while when evolutionary fractions are considered, we study the role of the evolutionary pressure, focusing on what happens with either identical or different marginal costs. Moreover, for the model with fixed fractions we investigate the effects of the production limiters and of the reaction speed. In such setting, we also show that marginal costs do not affect the local stability of the unique steady state, which coincides with the Nash equilibrium, and that its loss of stability occurs through a flip bifurcation. Furthermore, we prove that even if for most configurations the oligopoly size have a destabilizing effect, there exist oligopoly compositions which remain stable as the number of firms increases, differently from the other results about homogeneous oligopolies in the related literature.

For the evolutionary fractions model, we prove that there is a unique equilibrium fraction, which in general is not analytically computable. We then show that the effects of evolutionary pressure can change depending on the marginal costs difference. When the technology of the firms is identical or when the best response firms are more efficient than the rational ones, increasing the evolutionary pressure always leads to instability. Conversely, we provide simulative evidence of the fact that if the marginal cost of the rational firms is sufficiently smaller than the marginal cost of the best response firms, evolutionary pressure either can have no effect or may stabilize the dynamics. Such results, which are made possible by studying the effect of different marginal 
costs, are in contrast to the existing literature, in which evolutionary pressure only has a destabilizing role.

The remainder of the paper is organized as follows. In Section 2, we describe the static Cournot competition and the decisional mechanisms. In Section 3 we introduce and study the fixed fractions model. In Section 4 we deal with the evolutionary fractions model. Conclusions and future perspectives are collected in Section 5.

\section{Oligopolistic Cournot Game}

In the model we will deal with in the present paper, agents differ for their degree of rationality. In what follows we first introduce the static game; the decisional mechanisms are then described in Section 2.1.

We consider a homogeneous market characterized by $N$ firms that produce quantities $q_{i}, i=1, \ldots, N$, of the same good, for which we assume a linear inverse demand function

$$
p(Q)=a-b Q
$$

where $Q=\sum_{i=1}^{N} q_{i}$ is aggregate demand and $a, b$ are positive constants. In particular, in such formulation $a$ represents the market size. We assume that the firms have linear cost functions

$$
C\left(q_{i}\right)=c_{i} q_{i}
$$

where $c_{i} \in\left\{c_{1}, c_{2}\right\}, i=1, \ldots, N$, are two possibly different (constant) marginal costs. In such setting, the industry consists of two groups of $\omega N$ and $(1-\omega) N$ firms identical within each group, respectively, where $\omega$ represents the fraction of firms with marginal cost $c_{1}$.

The previous framework sets up a game, in which the players are the $N$ oligopolists, the admissible strategies are all the nonnegative production choices $q_{i} \geq 0$, and the payoff functions are the profit functions

$$
\pi_{i}=q_{i}(a-b Q)-c_{i} q_{i}, \quad i=1, \ldots, N .
$$

We only consider the situation in which all firms have nonnull production levels, and so we focus on the internal Nash equilibrium, namely, the equilibrium consisting of strictly positive strategies. We derive its expression in the next result.

\section{Proposition 1. Let}

$$
>\max \left\{c_{1}+N\left(c_{1}-c_{2}\right)(1-\omega), c_{2}-N \omega\left(c_{1}-c_{2}\right), 0\right\} .
$$

Then, there is a unique internal Nash equilibrium for our game, for which

$$
\begin{aligned}
& q_{1}^{\star}=\frac{a-c_{1}-N\left(c_{1}-c_{2}\right)(1-\omega)}{b(N+1)}, \\
& q_{2}^{\star}=\frac{a-c_{2}+N \omega\left(c_{1}-c_{2}\right)}{b(N+1)},
\end{aligned}
$$

where $q_{1}^{\star}$ is the equilibrium strategy of the firms having marginal cost $c_{1}$ and $q_{2}^{\star}$ is the equilibrium strategy of those having marginal cost $c_{2}$.
Proof. Firstly, we compute the best responses of the $i$ th player of the first group and of the $k$ th player of the second group with respect to the remaining players strategies. This can be done by deriving the first order conditions on payoffs of the two groups, obtaining

$$
\begin{aligned}
& a-b\left(Q_{1,-i}+Q_{2}\right)-2 b q_{1, i}-c_{1}=0 \\
& a-b\left(Q_{1}+Q_{2,-k}\right)-2 b q_{2, k}-c_{2}=0
\end{aligned}
$$

where $Q_{j}, j=1,2$, are the aggregate strategies of the players of the $j$ th group, and $Q_{1,-i}$ (resp., $Q_{2,-k}$ ) is the aggregate strategy of all the players belonging to the first (resp., second) group except the $i$ th (resp., $k$ th) player. From (6) we obtain the best response functions

$$
q_{1, i}=\frac{a-c_{1}-b\left(Q_{1,-i}+Q_{2}\right)}{2 b},
$$

provided that $a-c_{1}-b\left(Q_{1,-i}+Q_{2}\right)>0$, and

$$
q_{2, k}=\frac{a-c_{2}-b\left(Q_{1}+Q_{2,-k}\right)}{2 b},
$$

provided that $a-c_{2}-b\left(Q_{1}+Q_{2,-k}\right)>0$. It is easy to see that both (7) and (8) satisfy second order conditions. In order to obtain the equilibrium strategy by solving (7)-(8), we notice that players belonging to the same group are identical, so that they have the same equilibrium strategy

$$
\begin{aligned}
& q_{1, i}^{\star}=q_{1}^{\star}, \\
& q_{2, k}^{\star}=q_{2}^{\star},
\end{aligned}
$$

and, consequently,

$$
\begin{aligned}
& Q_{1}^{\star}=\omega N q_{1}^{\star}, \\
& Q_{2}^{\star}=(1-\omega) N q_{2}^{\star} .
\end{aligned}
$$

We obtain the internal Nash equilibrium (5a) and (5b) by inserting the previous expressions in (7) and (8) and solving the resulting $2 \times 2$ system, provided that (4) is satisfied.

We observe that in the Nash equilibrium it holds that $q_{1}^{*}>$ $q_{2}^{*}$ if and only if $c_{2}>c_{1}$.

2.1. The Decisional Mechanisms. In this work, we aim to study the dynamics arising when the two groups of firms adopt different decisional mechanisms. In particular, we will suppose that not all the firms are fully rational, so that a dynamic adjustment process naturally arises. The behavioral rules we focus on are best reply mechanisms with either perfect foresight (rational firms) or static expectations (best response firms). Rational firms have complete informational and computational capabilities, so that they are able to optimally respond to the other players strategies and are endowed with perfect foresight about the next period production level of their competitors. Best response firms have a reduced degree of rationality with respect to rational firms, as they assume their opponents next period production to be the same as in the past period. 
In the remainder of the present section we will obtain the reaction functions of each group of players. Without loss of generality, we assume that rational firms have marginal $\operatorname{cost} c_{1}$. Moreover, we denote by $Q_{1, t}$ the aggregate production of rational firms and by $Q_{2, t}$ the aggregate output of best response players, both at time $t$, while we will denote by $Q_{1,-i, t}$ the aggregate production at time $t$ of all rational firms except firm $i$. We remark that, in principle, both the best reply of rational and of best response players may be null. Since we only want to focus on positive dynamics for the production levels, we will not deal with such situations.

Rational Players. If we consider a generic rational firm $i$, we have that its profit is given by

$$
\begin{aligned}
\pi_{1, i}= & q_{1, i, t+1}\left(a-b\left(Q_{1,-i, t+1}+q_{1, i, t+1}+Q_{2, t+1}\right)\right) \\
& -c_{1} q_{1, i, t+1},
\end{aligned}
$$

where we denoted by $q_{1, i, t+1}$ the strategy of firm $i$ at time $t+1$, by $Q_{1,-i, t+1}$ the aggregate quantity at time $t+1$ of all the rational players but the $i$ th, and by $Q_{2, t+1}$ the aggregate strategy of the best response players at time $t+1$.

As noticed in the proof of Proposition 1, since all rational players are identical, we can compute the reaction function of a generic rational player with respect to the correctly foreseen strategies of the best response players.

Proposition 2. If $Q_{2, t+1}<\left(a-c_{1}\right) / b$, then the reaction function of a generic rational player $q_{1, i}$ at time $t+1$, one denotes by $q_{1, t+1}$, is given by

$$
q_{1, t+1}=\frac{a-c_{1}-b Q_{2, t+1}}{b(\omega N+1)} .
$$

Proof. We can compute the best response of the $i$ th rational player with respect to the aggregate strategies of the remaining rational players and of the best response players acting as in the proof of Proposition 1, obtaining from the first order conditions of (11)

$$
q_{1, i, t+1}=\frac{a-c_{1}-b\left(Q_{1,-i, t+1}+Q_{2, t+1}\right)}{2 b}
$$

provided that $a-c_{1}-b\left(Q_{1,-i, t+1}+Q_{2, t+1}\right)>0$. The second order conditions are indeed satisfied. Since all the rational players are identical, we can set $Q_{1,-i, t+1}=(\omega N-1) q_{1, i, t+1}$ in (13) and, solving the resulting equation with respect to $q_{1, i, t+1}$, we find (12), as desired.

Best Response Players. Best response players are able to optimally respond to the other players strategies, but since they do not have perfect foresight, they rather use static expectations. The reaction function of the generic $k$ th player at time $t+1$, we denote by $q_{2, k, t+1}$, is then the best response to $Q_{-k, t+1}$, which is the aggregate strategy at time $t+1$ of all the remaining players, namely,

$$
q_{2, k, t+1}=\frac{a-c_{2}-b Q_{-k, t}}{2 b}
$$

provided that $Q_{-k, t}<\left(a-c_{2}\right) / b$.

\section{Fixed Fractions Oligopoly Model}

Our purpose is to study a heterogeneous oligopoly, that is, a competition in which not all the firms adopt the same behavioral rule. To this end, we suppose that there is always at least one rational firm and at least one best response firm, namely, $\omega \in[1 / N, 1-1 / N]$. Moreover, we assume that the initial production levels of the best response firms are the same, which in particular means that their production levels are identical. Finally, since we only focus on economically interesting positive production levels, in what follows we consider initial data that give rise to positive trajectories. Such assumptions allow us to identify the generic rational and best response player's strategies, respectively, with

$$
\begin{aligned}
& q_{1, t}=q_{1, i, t}, \\
& q_{2, t}=q_{2, k, t},
\end{aligned}
$$

which give $Q_{1,-i, t}=(\omega N-1) q_{1, t}, Q_{2, t}=(1-\omega) N q_{2, t}$, and $Q_{-k, t}=\omega N q_{1, t}+((1-\omega) N-1) q_{2, t}$, and to rewrite (13) and (14) as

$$
\begin{aligned}
q_{1, t+1} & =\frac{a-c_{1}-b(1-\omega) N q_{2, t+1}}{b(\omega N+1)}, \\
q_{2, t+1} & =\frac{a-c_{2}-b\left(\omega N q_{1, t}+((1-\omega) N-1) q_{2, t}\right)}{2 b} .
\end{aligned}
$$

Combining (16) and (17) we have

$$
q_{2, t+1}=\frac{a-c_{2}-b\left(\omega N\left(\left(a-c_{1}-b(1-\omega) N q_{2, t}\right) /(b(\omega N+1))\right)+((1-\omega) N-1) q_{2, t}\right)}{2 b} .
$$

Gathering in the previous expression the terms with $q_{2, t}$, we obtain

$$
\begin{aligned}
& q_{2, t+1} \\
& =-b \frac{q_{2, t}}{2 b}\left(\frac{-b \omega(1-\omega) N^{2}}{b(\omega N+1)}+(1-\omega) N-1\right)
\end{aligned}
$$

$$
\begin{gathered}
+\frac{a-c_{2}-b \omega N\left(\left(a-c_{1}\right) /(b(\omega N+1))\right)}{2 b} \\
=-\frac{q_{2, t}}{2}\left(\frac{-\omega(1-\omega) N^{2}}{(\omega N+1)}+(1-\omega) N-1\right) \\
+\frac{a-c_{2}-\omega N\left(\left(a-c_{1}\right) /(\omega N+1)\right)}{2 b}
\end{gathered}
$$




$$
\begin{aligned}
= & -\frac{q_{2, t}}{2}\left(\frac{-\omega(1-\omega) N^{2}+((1-\omega) N-1)(\omega N+1)}{(\omega N+1)}\right) \\
& +\frac{\left(a-c_{2}\right)(\omega N+1)-\omega N\left(a-c_{1}\right)}{2 b(\omega N+1)} \\
= & -\frac{q_{2, t}}{2}\left(\frac{(1-\omega) N-(\omega N+1)}{(\omega N+1)}\right) \\
& +\frac{\left(a-c_{2}\right)+\omega N\left(c_{1}-c_{2}\right)}{2 b(\omega N+1)} \\
= & \frac{q_{2, t}}{2}\left(\frac{2 \omega N+2-1-N}{(\omega N+1)}\right)-\frac{\left(c_{2}-a\right)-\omega N\left(c_{1}-c_{2}\right)}{2 b(\omega N+1)},
\end{aligned}
$$

which gives

$$
\begin{aligned}
q_{2, t+1}= & f\left(q_{2, t}\right) \\
= & \left(1-\frac{(1+N)}{2(1+\omega N)}\right) q_{2, t} \\
& -\frac{c_{2}-a-\omega N\left(c_{1}-c_{2}\right)}{2 b(\omega N+1)} .
\end{aligned}
$$

However, we suppose that the best response player does not immediately choose the new production level $q_{2, t+1}$, but he cautiously moves towards it. This also reflects the fact that, in concrete situations, firms can only handle limited production variations, for example, because of capacity or financial constraints (see [31, page 507]), so that the maximum admissible upper and lower output variations may be described, by means of two positive parameters $a_{1}$ and $a_{2}$, as $a_{1}$ and $-a_{2}$, respectively. The possibility of varying $a_{1}$ and $a_{2}$ allows investigating the effects of the output limitation degree. The values assigned to such parameters depend on the kind of commodity under analysis and on its unit production time and are calibrated on the base of empirical research in different oligopolistic markets.

The cautious behavior of the best response player may be modeled in several ways, introducing, for instance, an inertial parameter which anchors the next time production to the strategy $q_{2, t}$, as done by Matsumoto and Szidarovszky in [5]. In our contribution, we adopt a different cautious adjustment, similar to that used by Du et al. in [31], which is obtained by (20) as follows:

$$
q_{2, t+1}=q_{2, t}+g\left(\gamma\left(f\left(q_{2, t}\right)-q_{2, t}\right)\right)
$$

where $g: \mathbb{R} \rightarrow \mathbb{R}$ is the nonlinear sigmoidal map defined as

$$
g(x)=a_{2}\left(\frac{a_{1}+a_{2}}{a_{1} e^{-x}+a_{2}}-1\right),
$$

with $a_{1}, a_{2}$ positive parameters, playing the role of horizontal asymptotes and restricting the possible output variation as, from period $t$ to $t+1, q_{2}$ can increase at most by $a_{1}$ and decrease at most by $a_{2}$. Indeed, $g$ is a strictly increasing function which takes values in $\left(-a_{2}, a_{1}\right)$ and, as shown in
Proposition 3, its introduction does not alter the steady states of (20). Parameter $\gamma>0$ in (21) represents the speed of reaction of the agents with respect to production level variations.

Variation limitation mechanisms similar to (21) have been also applied to different variables in macroeconomic settings, for instance, in $[32,33,37-40]$. As regards the specific shape of the output variation limiters in our work, instead of describing the output variation through a piecewise linear map, we chose to deal with the sigmoidal function in (22), which is differentiable and allows an easier mathematical treatment of the model. We stress that $S$-shaped functions alike to the one in (22) have already been considered, in a macroeconomic framework, in the Kaldorian business cycle model in [41], and, in a microeconomic setting, in the tâtonnement process in [42].

In view of the subsequent analysis, it is expedient to introduce the map $\varphi:(0,+\infty) \rightarrow \mathbb{R}$ associated with the dynamic equation in (21) and defined as

$$
\begin{aligned}
& \varphi\left(q_{2}\right)=q_{2}+g\left(\gamma\left(f\left(q_{2}\right)-q_{2}\right)\right)=q_{2} \\
& +a_{2}\left(\frac{a_{1}+a_{2}}{a_{1} e^{\gamma\left(((1+N) /(2(1+\omega N))) q_{2}+\left(c_{2}-a-N \omega\left(c_{1}-c_{2}\right)\right) /(2 b(N \omega+1))\right)}+a_{2}}\right. \\
& -1) .
\end{aligned}
$$

We remark that in the oligopolistic competition described by (21), the decisional mechanisms are exogenously assigned and do not change.

3.1. Stability and Bifurcation Analysis. We start studying the possible steady states of (21) and their connections with the Nash equilibrium. We have the following straightforward result.

Proposition 3. Equation (21) has a unique positive steady state which coincides with the Nash equilibrium (5a) and (5b).

Proof. The expression for $q_{2}^{\star}$ can be immediately found by solving the fixed point equation $\varphi(x)=x$, with $\varphi$ as in (23), noticing that no other solutions exist. The expression for $q_{1}^{\star}$ directly follows by that for $q_{2}^{\star}$ and by (16).

The next results are devoted to the study of the local stability of the Nash equilibrium. We also present several simulations to confirm the theoretical analysis and to investigate the loss of stability. As we are going to prove, marginal costs do not affect the stability, so in all the simulations we set $c_{1}=c_{2}=0.1$. Moreover, unless otherwise specified, we set $a_{1}=3, a_{2}=1$, and $\gamma=1$ in the sigmoid function in (22).

Lemma 4. For the dynamical equation in (21), the steady state $q_{2}^{\star}=\left(a-c_{2}+N \omega\left(c_{1}-c_{2}\right)\right) /(b(N+1))$ is locally asymptotically stable if

$$
\frac{\gamma a_{1} a_{2}(1+N)}{4\left(a_{1}+a_{2}\right)(1+\omega N)}<1 .
$$


Proof. A direct computation shows that

$$
\varphi^{\prime}\left(q_{2}^{\star}\right)=1-\frac{\gamma a_{1} a_{2}(1+N)}{2\left(a_{1}+a_{2}\right)(1+\omega N)} .
$$

Since $\varphi^{\prime}\left(q_{2}^{\star}\right)<1$ is always satisfied, the local asymptotic stability conditions require just that $\varphi^{\prime}\left(q_{2}^{\star}\right)>-1$, which is fulfilled for $\gamma a_{1} a_{2}(1+N) /\left(4\left(a_{1}+a_{2}\right)(1+\omega N)\right)<1$. This concludes the proof.

We conclude the present section by showing that when our dynamic equation loses stability at the steady state, a period-doubling bifurcation occurs.

Lemma 5. For the dynamical equation in (21), a perioddoubling bifurcation occurs at the steady state $q_{2}^{\star}=\left(a-c_{2}+\right.$ $\left.N \omega\left(c_{1}-c_{2}\right)\right) /(b(N+1))$ when $\gamma a_{1} a_{2}(1+N) /\left(4\left(a_{1}+a_{2}\right)(1+\right.$ $\omega N))=1$.

Proof. According to the proof of Proposition 1, the steady state $q_{2}^{*}$ is stable when $F^{\prime}\left(q_{2}^{*}\right)>-1$. Then, the map $F$ satisfies the canonical conditions required for a flip bifurcation (see [43]) and the desired conclusion follows. Indeed, when $F^{\prime}\left(Y^{*}\right)=-1$, that is, for $\gamma a_{1} a_{2}(1+N) /\left(4\left(a_{1}+a_{2}\right)(1+\omega N)\right)=$ 1 , then $Y^{*}$ is a nonhyperbolic fixed point; when $\gamma a_{1} a_{2}(1+$ $N) /\left(4\left(a_{1}+a_{2}\right)(1+\omega N)\right)<1$ it is attracting and finally when $\gamma a_{1} a_{2}(1+N) /\left(4\left(a_{1}+a_{2}\right)(1+\omega N)\right)>1$, it is repelling.

In the next corollaries, we will derive the stability conditions for $\gamma, \omega, N, a_{1}$, and $a_{2}$, respectively, in view of understanding whether they have a stabilizing or a destabilizing effect, and we will illustrate our conclusions through some pictures. We stress that those stability conditions may be immediately deduced by (4), by putting in evidence one of the parameters.

Corollary 6. For the dynamical equation in (21), the steady state $q_{2}^{\star}$ is locally asymptotically stable if

$$
\gamma<\frac{4\left(a_{1}+a_{2}\right)(1+\omega N)}{a_{1} a_{2}(1+N)} .
$$

Such result shows that, as expected, reactivity $\gamma$ has a destabilizing role. We illustrate such property in Figure 1, in which we consider an oligopoly of $N=50$ firms, with $\omega N=5$ rational firms, with an economy characterized by $a=30$ and $b=0.1$. The initial output level is set equal to $q_{2,0}=6$. In agreement with (26), equilibrium loses its stability for $\gamma \approx$ 0.6275 .

Corollary 7. For the dynamical equation in (21), the steady state $q_{2}^{\star}$ is locally asymptotically stable if

$$
\omega>\left(\frac{\gamma(1+N) a_{1} a_{2}}{4\left(a_{1}+a_{2}\right)}-1\right) \frac{1}{N} .
$$

Hence, increasing $\omega$, that is, the fraction of rational players, has a stabilizing role. However, if, for instance,

$$
\left(\frac{\gamma(1+N) a_{1} a_{2}}{4\left(a_{1}+a_{2}\right)}-1\right)>1,
$$

or, equivalently,

$$
N<\frac{8 a_{1}+8 a_{2}-a_{1} a_{2} \gamma}{a_{1} a_{2} \gamma},
$$

all the heterogeneous oligopoly configurations are stable.

For the simulation reported in Figure 2, we considered an oligopoly of $N=22$ firms, set in an economy characterized by $a=24$ and $b=0.2$. The initial strategy is $q_{2,0}=5$. The oligopoly composition is studied for $\omega \in[1 / 22,0.2]$. In the boxes, we highlight the regions corresponding to $1,2,3$, and 4 rational firms. Condition (27) predicts that the Nash equilibrium is stable for $\omega>0.1506$, and this means that at least 4 rational firms (i.e., $\omega=0.1818$ ) are needed to guarantee stability. We remark that with a reduced number of rational firms, we have both periodic and chaotic dynamics.

Moreover, we may put $N$ in evidence in (24), finding the following.

Corollary 8. For the dynamical equation in (21), the following conclusions hold true:

(1) if $\gamma a_{1} a_{2}-4\left(a_{1}+a_{2}\right)>0$, then the steady state $q_{2}^{\star}$ is never stable;

(2) if $\gamma a_{1} a_{2}-4 \omega\left(a_{1}+a_{2}\right)<0$, then $q_{2}^{\star}$ is always locally asymptotically stable;

(3) if $\gamma a_{1} a_{2}-4\left(a_{1}+a_{2}\right)<0<\gamma a_{1} a_{2}-4 \omega\left(a_{1}+a_{2}\right)$, then $q_{2}^{\star}$ is locally asymptotically stable for

$$
N<\frac{4\left(a_{1}+a_{2}\right)-\gamma a_{1} a_{2}}{\gamma a_{1} a_{2}-4 \omega\left(a_{1}+a_{2}\right)} .
$$

Hence, we can conclude that increasing the population size $N$ has overall a destabilizing effect. More precisely, Condition (1) says that, for sufficiently large reaction speeds, all the considered families of oligopolies, independently of their size and composition, have an unstable equilibrium. Conversely, there are oligopoly compositions (with $\omega>$ $\left(\gamma a_{1} a_{2}\right) /\left[4\left(a_{1}+a_{2}\right)\right]$, according to Condition (2)) which have a stable equilibrium independently of the oligopoly size. We stress that this is in contrast with the literature of homogeneous oligopolies, as there the oligopoly size has always a destabilizing role. In the present case, the neutral role of $N$ is possible thanks to the presence of rational players, on condition that their fraction does not change. In the remaining situations, oligopoly size has a destabilizing effect (see Condition (3)). We show this last situation in Figure 3, setting $a=20, b=0.1$ and considering oligopoly compositions with $\omega=1 / 9$. The initial strategy is $q_{2,0}=6.22$. As predicted by (30), only for oligopolies with size $N<$ 10.6364 the equilibrium is stable, so just the oligopoly with 9 best response firms and 1 rational firm has a stable steady state. In oligopolies with $N=18,27,36$ firms and 2, 3, 4 rational firms, respectively, we observe cyclic trajectories, while for $N=45$ and 5 rational firms the dynamics are chaotic.

Finally, putting in evidence $a_{1}$ or $a_{2}$ in (24) we obtain the following result. 

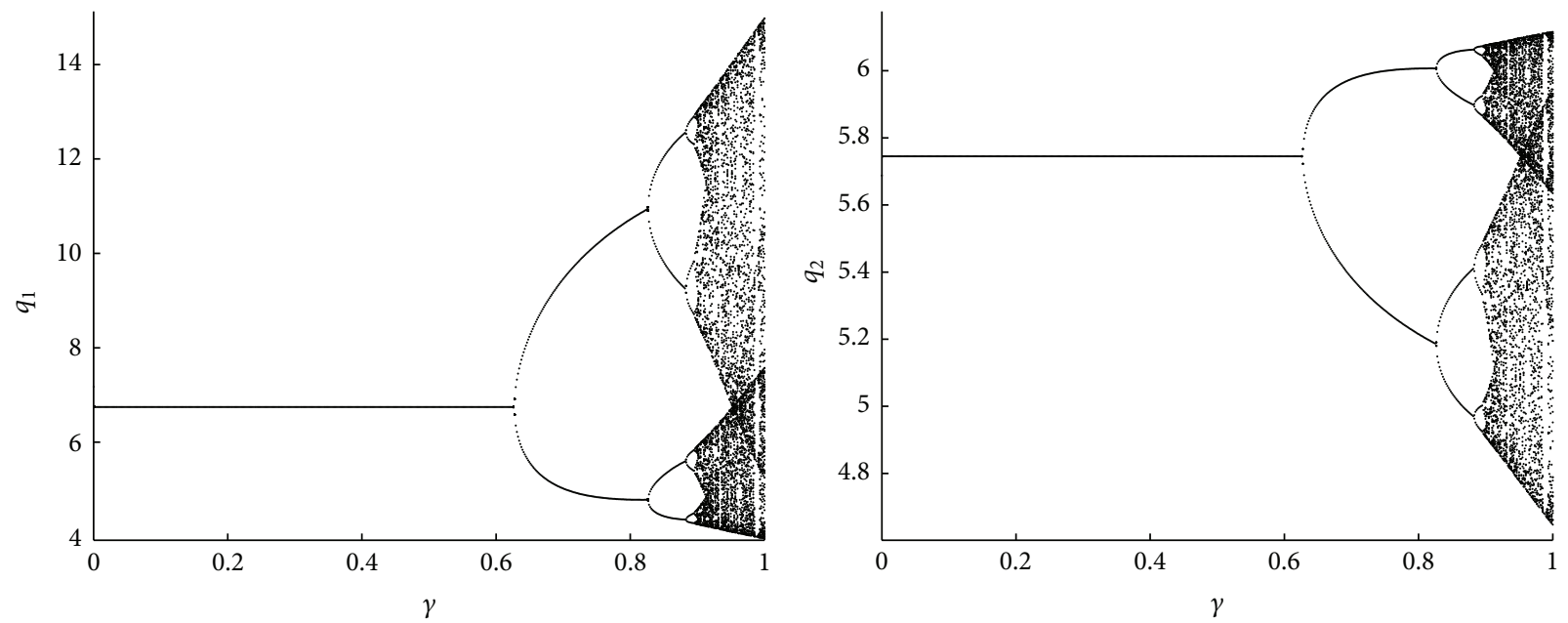

FIGURE 1: Bifurcation diagrams of quantities $q_{1}$ and $q_{2}$ with respect to the speed of reaction $\gamma$, which always has a destabilizing role.
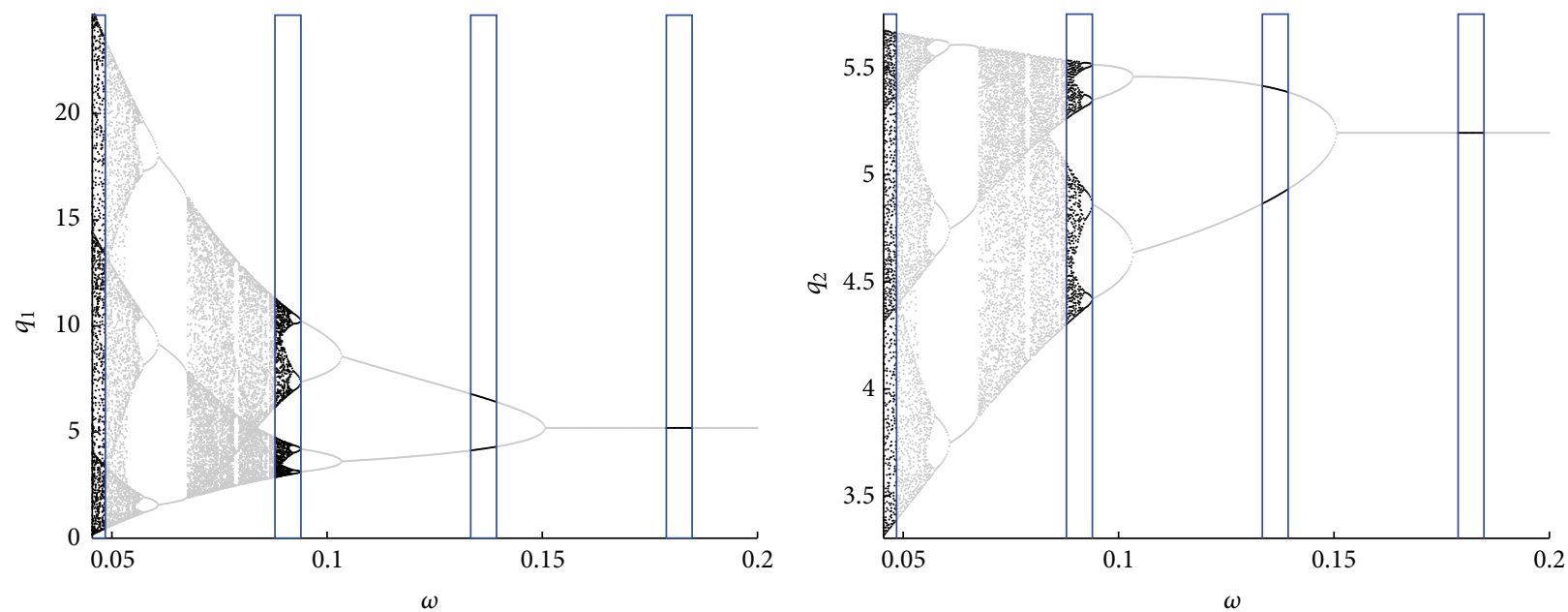

FIGURE 2: Bifurcation diagrams of quantities $q_{1}$ and $q_{2}$ with respect to the oligopoly composition $\omega$, for $N=22, a=24, b=0.2$. Increasing the number of best response firms leads to instability.
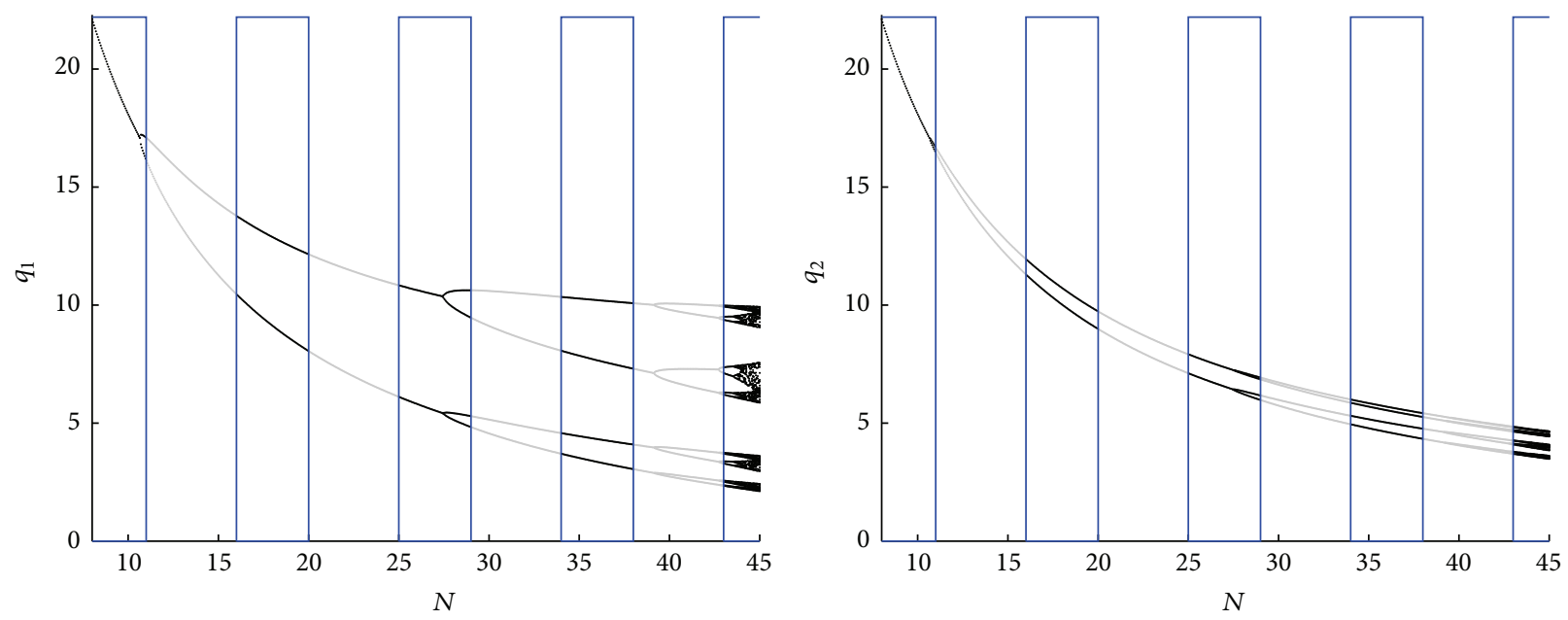

Figure 3: Bifurcation diagrams of quantities $q_{1}$ and $q_{2}$ with respect to the oligopoly size $N$, for $a=20, b=0.1$ and keeping fixed the oligopoly composition $\omega=1 / 9$. Increasing the oligopoly size has a destabilizing effect. 
Corollary 9. For the dynamical equation in (21), the following conclusions hold true:

(1) if $(1+N) a_{2} \gamma-4(1+\omega N)<0$, then the steady state $q_{2}^{\star}$ is always stable;

(2) if $(1+N) a_{2} \gamma-4(1+\omega N)>0$, then $q_{2}^{\star}$ is stable for

$$
a_{1}<\frac{4 a_{2}(1+\omega N)}{(1+N) a_{2} \gamma-4(1+\omega N)}
$$

(3) if $(1+N) a_{1} \gamma-4(1+\omega N)<0$, then $q_{2}^{\star}$ is always stable;

(4) if $(1+N) a_{1} \gamma-4(1+\omega N)>0$, then $q_{2}^{\star}$ is stable for

$$
a_{2}<\frac{4 a_{1}(1+\omega N)}{(1+N) a_{1} \gamma-4(1+\omega N)}
$$

Hence, from Conclusions (1) and (2) we can infer that increasing the upper bound $a_{1}$ has overall a destabilizing effect. In particular, an increase in $a_{1}$ is destabilizing when $-a_{2}$ is negative enough (see Figure 4 for a graphic illustration of the case with $N=15, \omega=1 / 3, a_{2}=3, a=$ $60, b=0.1, q_{0,2}=37.06$ ). Similarly, from Conclusions (3) and (4) it follows that decreasing the lower bound $-a_{2}$ has overall a destabilizing effect. In particular, a decrease in $-a_{2}$ is destabilizing when $a_{1}$ is large (see Figure 5 for a graphic illustration of the case with $N=15, \omega=1 / 3, a_{1}=3, a=$ $\left.60, b=0.1, q_{0,2}=37.06\right)$. We stress that the conclusions we have drawn from Corollary 9 may also be read in terms of chaos control, as they say that decreasing the output variation possibilities has a stabilizing effect.

\section{Endogenous Switching Mechanism}

We now assume that agents, in any time period, may switch between the groups of rational players and best response players. This requires introducing an evolutionary mechanism for the fractions, in which we will denote by $\omega_{t}$ the variable describing the fraction of rational players. To such end, we assume that the decisional rules are commonly known, as well as both past performances and costs. We assume that the firms first choose their decisional mechanism on the basis of the previous period performances, and then, according to such choice, they decide their strategy. Several switching mechanisms can be adopted. In the present work, in order to better model a form of competition in which each player is opposed to any other player, we adopt the logit choice rule, based on the logit function (see [34])

$$
z(x, y)=\frac{\exp (\beta x)}{\exp (\beta x)+\exp (\beta y)},
$$

where $x$ and $y$ denote the previous period performances by the two groups of firms and $z(x, y)$ describes the evolution of the fraction of firms whose past profits are given by $x$. Moreover, the positive parameter $\beta$ is usually referred to as the "evolutionary pressure" or "intensity of choice," and regulates the switching between the different decisional mechanisms, in the sense that as $\beta$ increases, it is more likely that firms switch to the decisional mechanism with higher net profits. Due to the supplementary degree of rationality of rational firms, we suppose that rational players incur costs for information gathering. We take into account such costs considering nonnull informational $\operatorname{costs} C>0$, so that the net profits of rational firms are actually given by $\pi_{1, t}=q_{1, t} p\left(Q_{t}\right)-$ $c_{1} q_{1, t}-C$.

Since we are interested in studying heterogeneous competitions only, we have to slightly modify the logit function, so that the switching variable assumes values just in $(1 / N,(N-$ $1) / N$ ) rather than in $[0,1]$. In this way, we always have at least a firm for each kind of decisional mechanism. Hence, we are led to consider the following two-dimensional dynamical system:

$$
\begin{aligned}
& q_{2, t+1}=q_{2, t} \\
& \quad+a_{2}\left(\frac{a_{1}+a_{2}}{a_{1} e^{\left.\left.\gamma((1+N)) /\left(2\left(1+\omega_{t} N\right)\right)\right) q_{2, t}+\left(c_{2}-a-N \omega_{t}\left(c_{1}-c_{2}\right)\right) /\left(2 b\left(N \omega_{t}+1\right)\right)\right)}+a_{2}}\right. \\
& \quad-1), \\
& \omega_{t+1}=\frac{1}{N}+\frac{N-2}{N} \frac{1}{1+e^{\beta \Delta \pi_{t}}},
\end{aligned}
$$

where the net profit differential is

$$
\begin{aligned}
\Delta \pi_{t} & =\pi_{2, t}-\pi_{1, t} \\
& =q_{2, t}\left(a-b Q_{t}-c_{2}\right)-q_{1, t}\left(a-b Q_{t}-c_{1}\right)+C,
\end{aligned}
$$

with

$$
q_{1, t}=\frac{a-c_{1}-b\left(1-\omega_{t}\right) N q_{2, t}}{b\left(\omega_{t} N+1\right)}
$$

and thus

$$
Q_{t}=\frac{N\left(\omega_{t}\left(a-c_{1}\right)+q_{2, t} b\left(1-\omega_{t}\right)\right)}{b\left(\omega_{t} N+1\right)} .
$$

We stress that in this way we are assuming that the rational players also know which will be the next period oligopoly composition $\omega_{t+1}$, while the best response players expect it to remain the same as in the previous period.

Notice that when $\beta=0$ the evolutionary mechanism reduces to $\omega_{t+1}=1 / 2$, and thus, for any initial partitioning of the population, the players do not consider nor compare the performances but they immediately split uniformly between the two decisional mechanisms. Conversely, as $\beta \rightarrow \infty$, the logit function becomes similar to a step function, so that the firms are inclined to rapidly switch to the most profitable mechanism and $\omega_{t+1}$ approaches $1 / N$ or $1-1 / N$. In particular, if the net profit differential is positive (i.e., best response firms achieve the best profits), the number of best response firms will be larger than that of the rational players $\left(\omega_{t+1}>1 / 2\right)$ and vice versa $\left(\omega_{t+1}<1 / 2\right)$ when the profit differential is negative.

We start by analyzing the simpler case in which the marginal costs $c_{1}$ and $c_{2}$ coincide, and then we will investigate the general framework with $c_{1} \neq c_{2}$.

(i) The Case with $c_{1}=c_{2}$. In this particular framework in which we assume that the marginal costs for all firms 

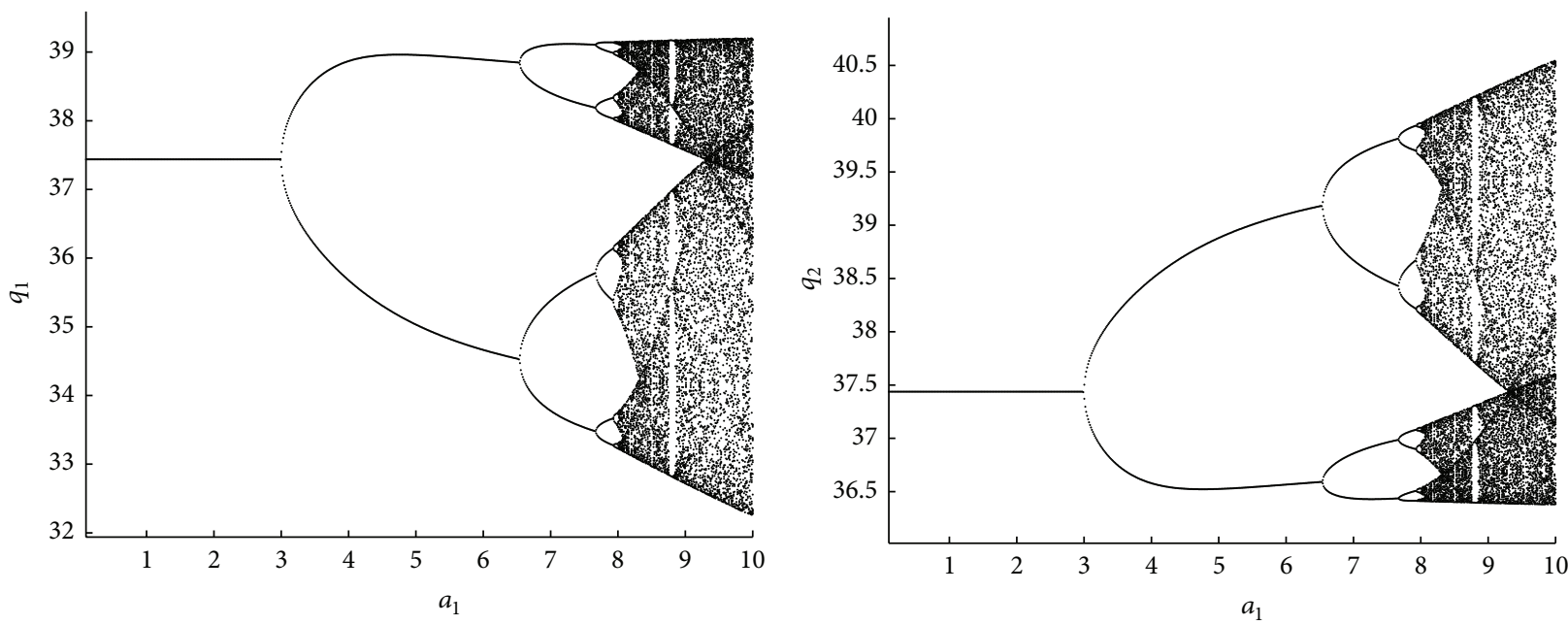

FIGURE 4: Bifurcation diagrams of quantities $q_{1}$ and $q_{2}$ with respect to the upper bound of production variation $a_{1}$. Increasing $a_{1}$ leads to instability.
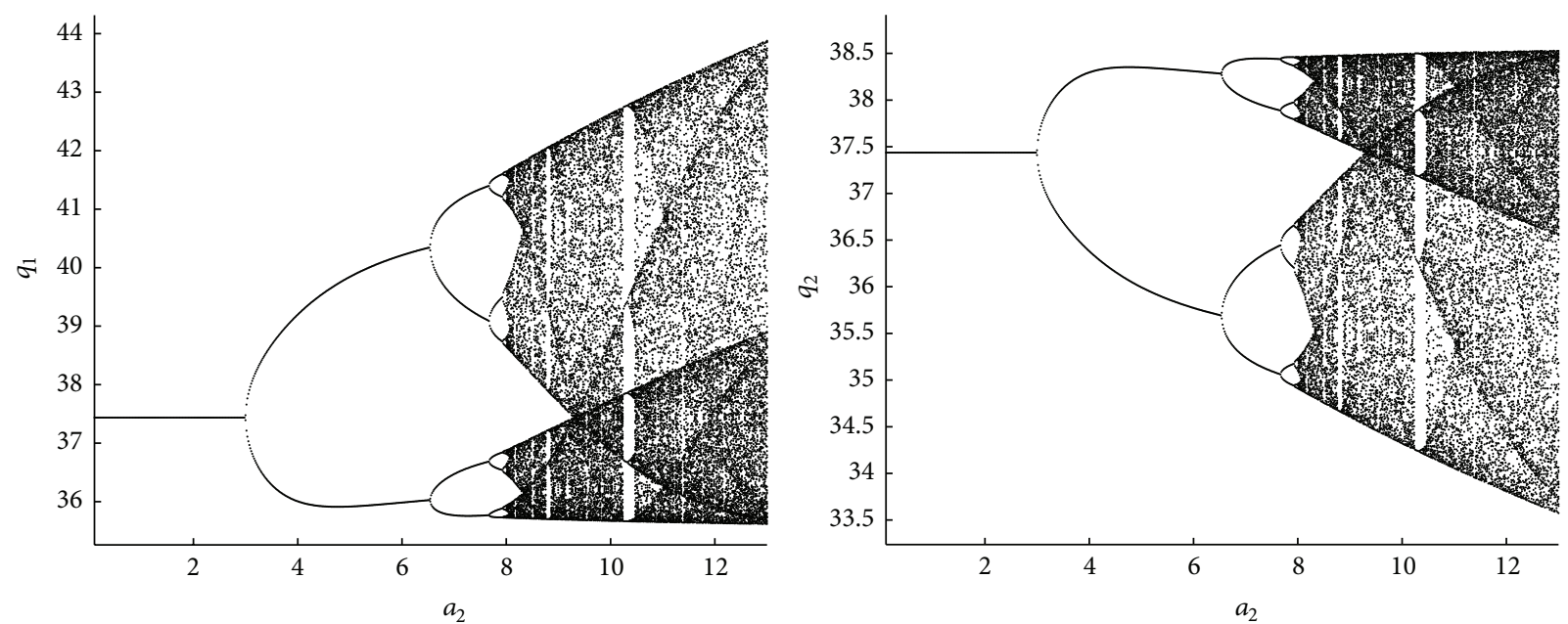

FIGURE 5: Bifurcation diagrams of quantities $q_{1}$ and $q_{2}$ with respect to the lower bound of production variation $-a_{2}$. Increasing $a_{2}$, namely, decreasing the lower bound $-a_{2}$, leads to instability.

coincide, and we set $c_{1}=c_{2}=c$, it is possible to analytically find the unique solution to system (34). Indeed, since by (5a) and (5b) it follows that

$$
q_{1}^{*}=q_{2}^{*}=\frac{a-c}{b(N+1)},
$$

then in equilibrium $\Delta \pi^{*}=\pi_{2}^{*}-\pi_{1}^{*}=q_{2}^{*}\left(a-b Q^{*}-c\right)-q_{1}^{*}(a-$ $\left.b Q^{*}-c\right)+C=C$ and thus

$$
\omega^{*}=\frac{1}{N}\left(1+\frac{N-2}{1+e^{\beta C}}\right) .
$$

Notice that the expression for $q_{1}^{*}=q_{2}^{*}$ in (38) does not depend on $\omega$ and that such steady state values are positive for $a>c$ (we stress that a large enough value for $a$ makes the steady state values for $q_{1}$ and $q_{2}$ positive also in the general case with possibly different marginal costs (see (5a) and (5b))). Hence, we will maintain such assumption in the remainder of the paper.
The stability analysis requires computing at the steady state $\left(q_{2}^{*}, \omega^{*}\right)$ the Jacobian matrix for the bidimensional function

$$
\begin{aligned}
& G:(0,+\infty) \times\left[\frac{1}{N}, \frac{N-1}{N}\right] \longrightarrow \mathbb{R} \times\left(\frac{1}{N}, \frac{N-1}{N}\right), \\
& \left(q_{2}, \omega\right) \longmapsto\left(G_{1}\left(q_{2}, \omega\right), G_{2}\left(q_{2}, \omega\right)\right), \\
& G_{1}\left(q_{2}, \omega\right)=q_{2} \\
& \quad+a_{2}\left(\frac{a_{1}+a_{2}}{a_{1} e^{\gamma\left(((1+N) /(2(1+\omega N))) q_{2}+\left(c_{2}-a-N \omega\left(c_{1}-c_{2}\right)\right) /(2 b(N \omega+1))\right)}+a_{2}}\right. \\
& \quad-1), \\
& G_{2}\left(q_{2}, \omega\right)=\frac{1}{N}\left(1+\frac{N-2}{1+e^{\beta \Delta \pi}}\right),
\end{aligned}
$$

where

$$
\Delta \pi=\pi_{2}-\pi_{1}=\left(q_{2}-q_{1}\right)(a-b Q-c)+C,
$$


with $q_{1}=\left(a-c_{1}-b(1-\omega) N q_{2}\right) /(b(\omega N+1))$ and thus $Q=$ $N\left(\omega(a-c)+q_{2} b(1-\omega)\right) /(b(\omega N+1))$.

It holds that

$$
J_{G}\left(q_{2}^{*}, \omega^{*}\right)=\left[\begin{array}{cc}
1-\frac{\gamma a_{1} a_{2}(1+N)}{2\left(a_{1}+a_{2}\right)\left(1+\omega^{*} N\right)} & 0 \\
J_{21} & 0
\end{array}\right],
$$

where $J_{21}=\partial_{q_{2}} G_{2}\left(q_{2}^{*}, \omega^{*}\right)$ is not essential in view of the subsequent computations. Indeed, the standard Jury conditions (see [44]) for the stability of our system read as

$$
\begin{aligned}
1- & \operatorname{det} J_{G}\left(q_{2}^{*}, \omega^{*}\right)=1>0, \\
1+ & \operatorname{tr} J_{G}\left(q_{2}^{*}, \omega^{*}\right)+\operatorname{det} J_{G}\left(q_{2}^{*}, \omega^{*}\right) \\
& =1+1-\frac{\gamma a_{1} a_{2}(1+N)}{2\left(a_{1}+a_{2}\right)\left(1+\omega^{*} N\right)}>0, \\
1- & \operatorname{tr} J_{G}\left(q_{2}^{*}, \omega^{*}\right)+\operatorname{det} J_{G}\left(q_{2}^{*}, \omega^{*}\right) \\
= & 1-1+\frac{\gamma a_{1} a_{2}(1+N)}{2\left(a_{1}+a_{2}\right)\left(1+\omega^{*} N\right)}>0 .
\end{aligned}
$$

Notice that just the second one is not always satisfied, but it leads to (24), in which we have to insert the expression for $\omega^{*}$ from (39). Then it may be rewritten as

$$
e^{\beta C}<\frac{4\left(a_{1}+a_{2}\right) N-\gamma a_{1} a_{2}(1+N)}{a_{1} a_{2}(1+N) \gamma-8\left(a_{1}+a_{2}\right)} .
$$

Since $\beta C>0$, the latter relation is never satisfied when the right hand side is smaller than 1 , that is, when

$$
\frac{4\left(a_{1}+a_{2}\right)(N+2)-2 \gamma a_{1} a_{2}(1+N)}{a_{1} a_{2}(1+N) \gamma-8\left(a_{1}+a_{2}\right)}<0,
$$

which is fulfilled for $\gamma<\underline{\gamma}$ and for $\gamma>\bar{\gamma}$, with

$$
\begin{aligned}
& \underline{\gamma}=\frac{8\left(a_{1}+a_{2}\right)}{a_{1} a_{2}(1+N)}, \\
& \bar{\gamma}=\frac{2\left(a_{1}+a_{2}\right)(N+2)}{a_{1} a_{2}(1+N)} .
\end{aligned}
$$

Notice that $\bar{\gamma}>\gamma$ when $N>2$. When $N=2$, (45) reads as $-2<0$, which is true for any value of $\gamma$.

In the remaining cases, that is, when the right hand side of (44) is larger than 1, the latter is fulfilled for

$$
\beta C<\log \left(\frac{4\left(a_{1}+a_{2}\right) N-\gamma a_{1} a_{2}(1+N)}{a_{1} a_{2}(1+N) \gamma-8\left(a_{1}+a_{2}\right)}\right) .
$$

The above considerations say that when the marginal costs are identical, the evolutionary pressure has a destabilizing effect. Such phenomenon is illustrated by the simulation in Figure 6, in which we considered an oligopoly of $N=100$ firms in an economy described by $a=120$ and $b=0.1$. Marginal costs are $c_{1}=c_{2}=0.2$ and the informational cost of the rational firm is $C=0.1$. The initial strategy is $q_{2,0}=9$ and the initial fraction of rational firms is $\omega_{0}=0.1$. Moreover, we set $a_{1}=1, a_{2}=3$, and $\gamma=0.34$. Since the steady state value for the fraction of rational firms is decreasing in Figure 6, firms are likely to switch to the best response rule, which gives larger profits. When $\beta C$ increases and reaches the value 3.2, the equilibrium loses its stability through a flip bifurcation.

(ii) The Case with $c_{1} \neq c_{2}$. When the marginal costs are different, the expression for the steady state value for $\omega$ for (34) can not be analytically computed, but a simple continuity argument shows that a steady state for system (34) always exists and that it is unique. Indeed, at any steady state $\left(q_{2}^{\star}, \omega^{\star}\right)$ it holds that, similarly to what obtained in (5b) for the case without switching mechanism,

$$
q_{2}^{\star}=\frac{a-c_{2}+N \omega^{\star}\left(c_{1}-c_{2}\right)}{b(N+1)} .
$$

Let us then define the continuous map

$$
\begin{aligned}
\psi:\left[\frac{1}{N}, \frac{N-1}{N}\right] & \longrightarrow \mathbb{R}, \\
\omega & \longmapsto \frac{1}{N}\left(1+\frac{N-2}{1+e^{\beta \Delta \pi(\omega)}}\right),
\end{aligned}
$$

where in the expression

$$
\begin{aligned}
\Delta \pi(\omega)= & \left(\pi_{2}-\pi_{1}\right)(\omega) \\
= & q_{2}(\omega)\left(a-b Q(\omega)-c_{2}\right) \\
& -q_{1}(\omega)\left(a-b Q(\omega)-c_{1}\right)+C,
\end{aligned}
$$

with

$$
\begin{aligned}
& q_{2}(\omega)=\frac{a-c_{2}+N \omega\left(c_{1}-c_{2}\right)}{b(N+1)}, \\
& q_{1}(\omega)=\frac{a-c_{1}-b(1-\omega) N q_{2}(\omega)}{b(\omega N+1)},
\end{aligned}
$$

and thus

$$
Q(\omega)=\frac{N\left(\omega\left(a-c_{1}\right)+q_{2}(\omega) b(1-\omega)\right)}{b(\omega N+1)},
$$

we wish to underline the dependence of all terms on $\omega$.

Since $(N-2) /\left(1+e^{\beta \Delta \pi(\omega)}\right)$ ranges in $(0, N-2)$, then $\psi(\omega)$ ranges in $(1 / N,(N-1) / N)$. Hence, $\psi$ is a continuous function that maps $[1 / N,(N-1) / N]$ into $(1 / N,(N-1) / N)$ and thus, by the Brouwer fixed point theorem, there exists (at least one) $\omega^{*} \in(1 / N,(N-1) / N)$ such that $\psi\left(\omega^{*}\right)=\omega^{*}$; that is, $\omega^{*}$ is (a) steady state value for system (34) together with $q_{2}^{\star}$ in (48). The value of the steady state for $q_{1}$ can finally be found by (36).

Let us now prove that system (34) admits a unique steady state. To such aim, it suffices to show that $\Delta \pi(\omega)$ in (50) is monotone with respect to $\omega$. In particular, we will show that the derivative of $\Delta \pi(\omega)$ with respect to $\omega$, we denote by $(\Delta \pi)^{\prime}(\omega)$, is always positive. Indeed, cumbersome but trivial computations leads to $(\Delta \pi)^{\prime}(\omega)=2 N\left(c_{1}-c_{2}\right)^{2} / b(N+1)>0$. This concludes the proof.

Finally, we report the expression for the Jacobian matrix at $\left(q_{2}^{*}, \omega^{*}\right)$ for the bidimensional function associated with 

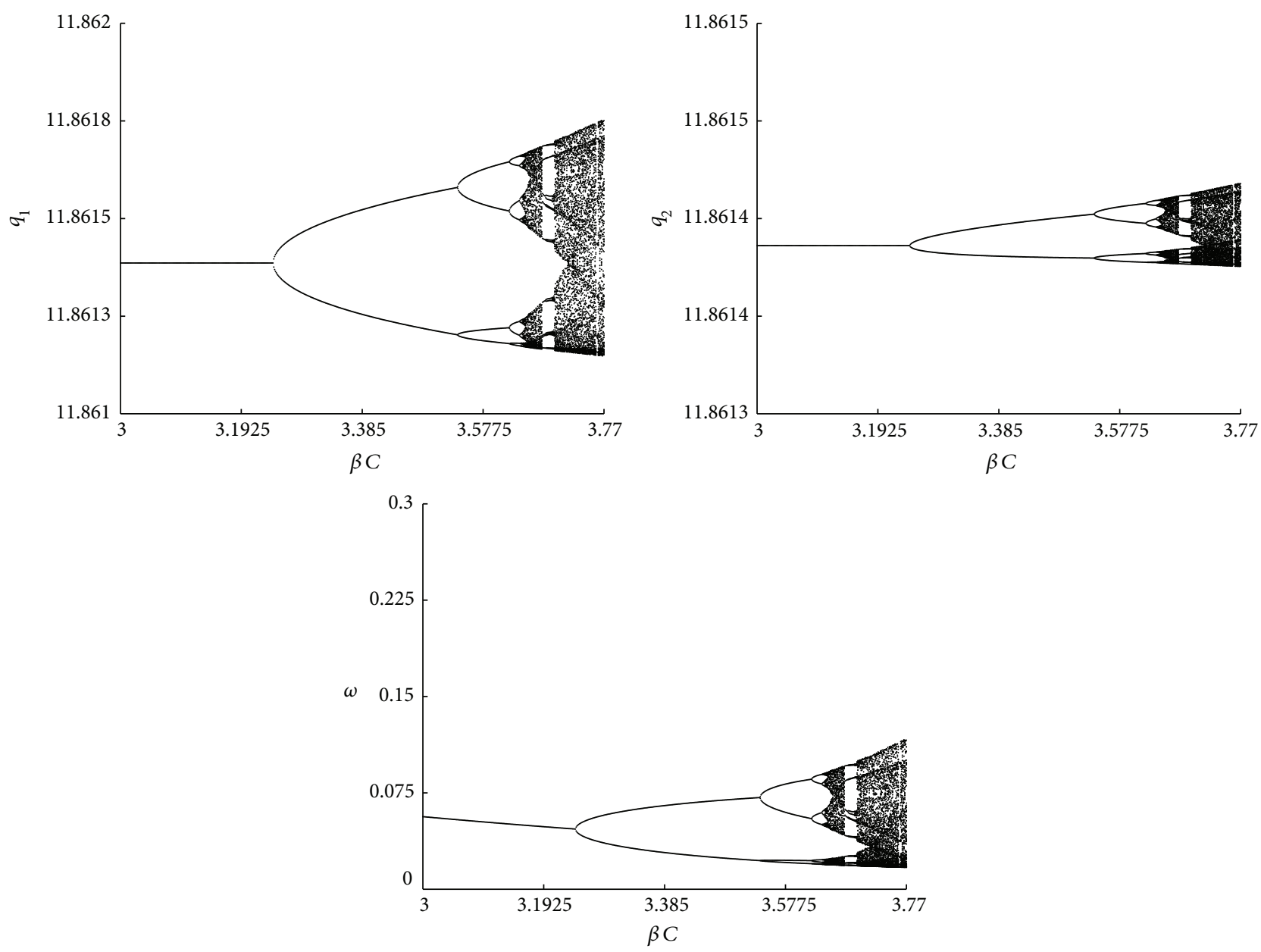

Figure 6: Bifurcation diagrams of quantities $q_{1}$ and $q_{2}$ and fraction $\omega$ with respect to the evolutionary pressure $\beta$. Increasing $\beta$ leads to instability.

(34), we still denote by $G$, due to the similarity with the case with equal marginal costs (see (40)), omitting the (tedious) computations leading to its derivation. Of course, in this case we do not know the value of the steady state $\left(q_{2}^{*}, \omega^{*}\right)$. Hence, we will just use (48) in order to express $q_{2}^{*}$ as a function of $\omega^{*}$.

It holds that

$$
\begin{aligned}
& J_{G}\left(q_{2}^{*}, \omega^{*}\right) \\
& =\left[\begin{array}{cc}
1-\frac{\gamma a_{1} a_{2}(1+N)}{2\left(a_{1}+a_{2}\right)\left(1+\omega^{*} N\right)} & \frac{\gamma a_{1} a_{2} N\left(c_{1}-c_{2}\right)}{2 b\left(a_{1}+a_{2}\right)\left(1+\omega^{*} N\right)} \\
J_{21} & J_{22}
\end{array}\right],
\end{aligned}
$$

where

$$
\begin{aligned}
& J_{21} \\
& =-\frac{\beta(N-2)\left(a-c_{2}-2 N c_{1}+2 N c_{2}+3 N c_{1} \omega^{*}-3 N c_{2} \omega^{*}\right) e^{\beta \Delta \pi}}{N\left(1+e^{\beta \Delta \pi}\right)^{2}\left(N \omega^{*}+1\right)},
\end{aligned}
$$

or, since in equilibrium we have

$$
\omega^{*}=\frac{1}{N}\left(1+\frac{N-2}{1+e^{\beta \Delta \pi}}\right)
$$

equivalently

$$
\begin{aligned}
& J_{21}=-\frac{\beta\left(N \omega^{*}-1\right)\left(N-N \omega^{*}-1\right)\left(a-c_{2}-2 N c_{1}+2 N c_{2}+3 N c_{1} \omega^{*}-3 N c_{2} \omega^{*}\right)}{N(N-2)\left(N \omega^{*}+1\right)}, \\
& J_{22}=-\frac{\beta(N-2)\left(c_{1}-c_{2}\right)\left(2 c_{1}-a-c_{2}+2 N c_{1}-2 N c_{2}-N c_{1} \omega^{*}+N c_{2} \omega^{*}\right) e^{\beta \Delta \pi}}{b\left(1+e^{\beta \Delta \pi}\right)^{2}\left(N \omega^{*}+1\right)(N+1)},
\end{aligned}
$$


or equivalently, using again (55),

$$
\begin{aligned}
& J_{22} \\
& =-\frac{\beta\left(N \omega^{*}-1\right)\left(N-N \omega^{*}-1\right)\left(c_{1}-c_{2}\right)}{b(N-2)\left(N \omega^{*}+1\right)(N+1)} \\
& \quad \cdot\left(2 c_{1}-a-c_{2}+2 N c_{1}-2 N c_{2}-N c_{1} \omega^{*}+N c_{2} \omega^{*}\right) .
\end{aligned}
$$

The elements of $J_{G}\left(q_{2}^{*}, \omega^{*}\right)$ are too complicated and do not allow obtaining general stability conditions. Hence, in order to investigate the possible scenarios arising when the marginal costs are different, we focus on the following example. We set $a=100, b=0.1$ for the economy, $N=$ $100, c_{1}=0.1, C=0.05$ for the firms, $a_{1}=1, a_{2}=$ $3, \gamma=0.34$ for the decisional mechanism and we study what happens for different values of the marginal costs of best response firms. We consider both the frameworks with $c_{2}<c_{1}$ and $c_{1}<c_{2}$, giving special attention to the latter case, as it describes the economically interesting situation in which rational firms have improved technology (i.e., reduced marginal costs) at the price of larger fixed costs.

We start by considering the setting with $c_{2}=0.101$, so that the marginal costs of the best response firms is larger but very close to that of the rational firms. In this case, the profit differential at the equilibrium is

$$
\Delta \pi\left(q_{2}^{*}, \omega^{*}\right)=\frac{\omega^{*}}{50500}+\frac{305101}{10100000}>0
$$

We stress that when the net profit differential is positive, the possible equilibrium fractions are only $\omega^{*} \in(1 / N, 1 / 2)$. Moreover, the positivity of (58) implies that increasing $\beta$, $\omega^{\star}$ decreases and thus the fraction of rational players may become too small to guarantee stability. This conjecture is confirmed by Figure 7 , where we can see that for $\beta \approx 68.19$ the equilibrium loses its stability through a flip bifurcation. In fact, for $\beta \approx 65.19$ the equilibrium fraction can be computed numerically and is given by $\omega^{*}=0.13$ (corresponding to 13 rational firms out of 100) which implies the equilibrium strategy $q_{2}^{*}=9.8897$. The largest eigenvalue of $J_{G}\left(q_{2}^{*}, \omega^{*}\right)$ that corresponds to this configuration is such that $|\lambda|=0.9287$, which means that the equilibrium is stable. Conversely, for $\beta=68.45$, the equilibrium fraction is $\omega^{*}=0.12$, which gives the equilibrium strategy $q_{2}^{*}=9.889789$ and the eigenvalues of $J_{G}\left(q_{2}^{*}, \omega^{*}\right)$ are $\lambda_{1}=0.506$ and $\lambda_{2}=-1.0006$; that is, equilibrium has lost its stability.
If we further increase the marginal cost of the best response firms, setting, for example, $c_{2}=0.11$, we find that the equilibrium is stable independently of the evolutionary pressure. In fact, in this case, we have that the profit differential is given by

$$
\Delta \pi\left(q_{2}^{*}, \omega^{*}\right)=\frac{\omega^{*}}{505}-\frac{15029}{101000}
$$

which is negative for all the possible $\omega^{*} \in[1 / N, 1-$ $1 / N)$. In this case, since the equilibrium fraction increases with $\beta$, namely, the fraction of rational players increases, we can expect the stability not to be affected by evolutionary pressure. To prove such conjecture, we notice that at the equilibrium we have

$$
\omega^{*}=\frac{49}{50\left(e^{\beta\left(200 \omega^{*}-15029\right) / 101000}+1\right)}+\frac{1}{100} \geq \frac{1}{2}
$$

from which we can obtain the evolutionary pressure $\beta$ to which the equilibrium fraction $\omega^{*}$ corresponds; that is,

$$
\beta=\frac{\log \left(\left(99-100 \omega^{*}\right) /\left(100 \omega^{*}-1\right)\right)}{\omega^{*} / 505-15029 / 101000}
$$

Now we consider Jury conditions (43). After substituting the parameter values and using (61), the first condition reduces to

$$
\begin{aligned}
& L\left(\omega^{*}\right) \\
& \quad \cdot \frac{\left(100 \omega^{*}-1\right)\left(99-100 \omega^{*}\right)\left(2033049-20000 \omega^{*}\right)}{19600\left(100 \omega^{*}+1\right)\left(15029-200 \omega^{*}\right)} \\
& \quad<1,
\end{aligned}
$$

where we set

$$
L\left(\omega^{*}\right)=\log \left(\frac{99-100 \omega^{*}}{100 \omega^{*}-1}\right)
$$

Condition (62) is satisfied, since $L\left(\omega^{*}\right) \leq 0$ as the argument of the logarithm is not larger than 1 for $\omega^{*} \geq 1 / 2$ and the second factor in (62) is indeed positive for $\omega^{*} \in[1 / 2,1-1 / N)$.

The second condition in (43) reduces to

$$
\frac{\left(99-100 \omega^{*}\right)\left(\omega^{*}-0.01\right)\left(40000 \omega^{*}-4071249\right) L\left(\omega^{*}\right)+3959200000 \omega^{*}-215330990}{19796000\left(100 \omega^{*}+1\right)}>0,
$$

and the positivity of the 1.h.s. for $\omega^{*} \in[1 / 2,1-1 / N)$ can be studied using standard analytical tools. We avoid entering into details and we only report in Figure 8 the plot of the function corresponding to the 1.h.s. of (64) with respect to $\omega^{*}$, which shows its positivity.
The last condition in (43) reads as

$$
\begin{aligned}
& \frac{\left(-510000\left(\omega^{*}\right)^{2}+510000 \omega^{*}-5049\right) L\left(\omega^{*}\right)+252399000}{1960000000 \omega^{*}+19600000} \\
& >0 .
\end{aligned}
$$



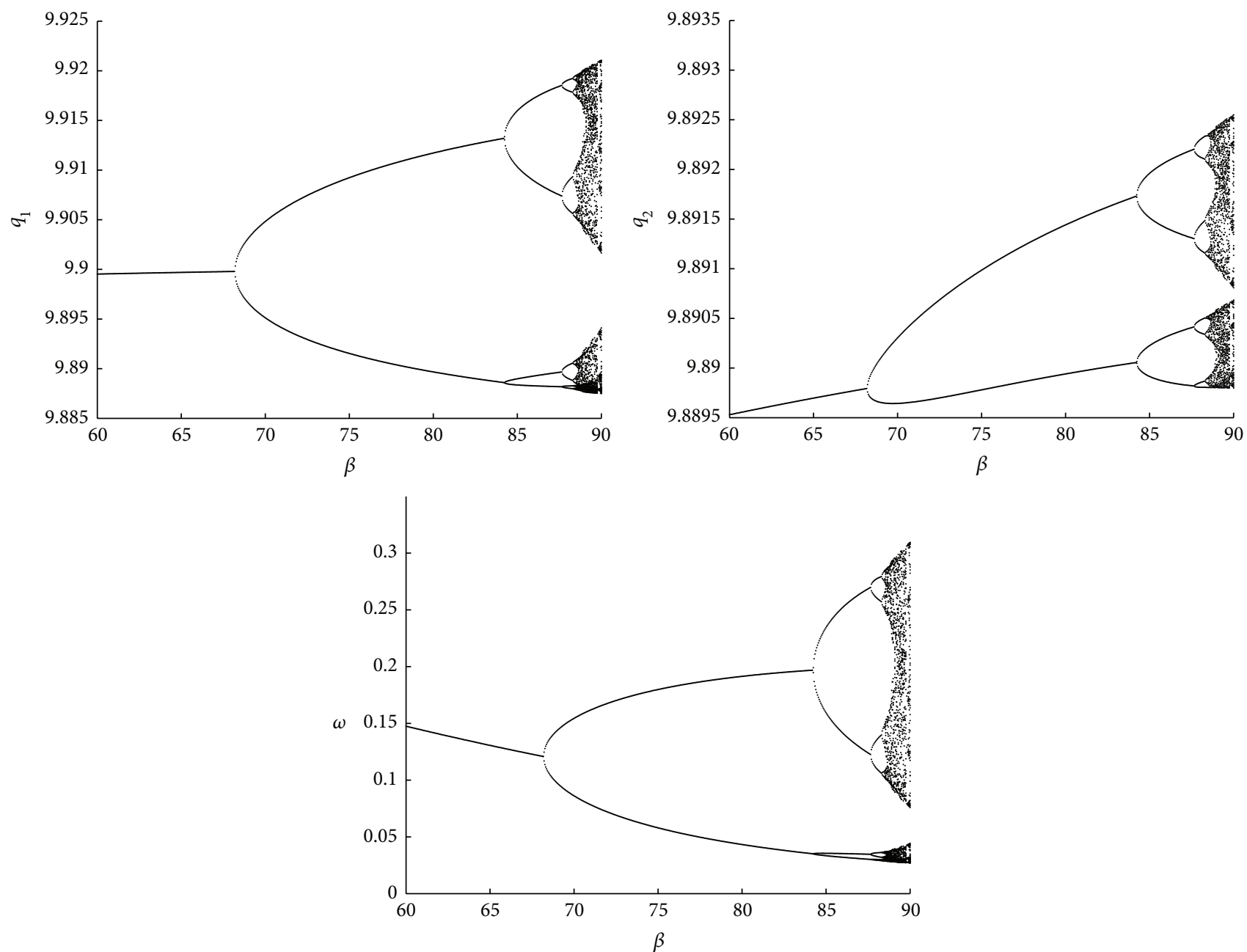

Figure 7: Bifurcation diagrams of quantities $q_{1}$ and $q_{2}$ and fraction $\omega$ with respect to the evolutionary pressure $\beta$. Increasing $\beta$ has a destabilizing effect for $c_{2}$ slightly larger than $c_{1}$.

Like for the second condition, we omit the analytical study of the l.h.s. of (65) and we only report the plot of the corresponding function in Figure 9, which shows the positivity. Then, each stability condition is satisfied for all $\beta$, which proves that, at least for this parameter configuration, the equilibrium is stable independently of the evolutionary pressure.

Then, we consider $c_{1}>c_{2}=0.09$, case in which, as one might expect, evolutionary pressure has a destabilizing effect. In fact, we have that the net profit differential

$$
\Delta \pi\left(q_{2}^{*}, \omega^{*}\right)=\frac{\omega^{*}}{505}+\frac{24931}{101000}
$$

is positive independently of $\omega^{*}$. The resulting scenario is analogous to the first one, with bifurcation diagrams qualitatively similar to those reported in Figure 7. The flip bifurcation occurs for $\beta \approx 16.252$. In this case, for $\beta=15.68$, the equilibrium fraction can be computed numerically and is given by $\omega^{*}=0.03$ (3 rational firms out of 100 ), which implies the equilibrium strategy $q_{2}^{*}=9.895$. The largest eigenvalue of $J_{G}\left(q_{2}^{*}, \omega^{*}\right)$ is such that $|\lambda|=0.864<1$ and thus the equilibrium is stable.
Conversely, the equilibrium fraction corresponding to $\beta=18.53$ is $\omega^{*}=0.02$ (2 rational firms out of 100 ), which gives the equilibrium strategy $q_{2}^{*}=9.894$. The eigenvalues of $J_{G}\left(q_{2}^{*}, \omega^{*}\right)$ are now $\lambda_{1}=-0.2$ and $\lambda_{2}=-2.4$, and thus the equilibrium is no more stable.

A question naturally arises from the second example: what happens if the configuration corresponding to $\beta=0$ is unstable and the marginal cost is sufficiently favorable to the rational firms? Let us consider, for instance, the previous parameters for the economy and the firms, but $a_{1}=100, a_{2}=$ 3, $\gamma=0.94$ for the decisional mechanism of the best response firms. We underline that the new value for $a_{1}$ allows a larger increase in production, while the increased $\gamma$ models firms which are more reactive to production variations. The general scenario is indeed more "unstable." If we take $c_{2}=0.18$, the behavior for increasing values of the reaction speed is reported in the bifurcation diagrams of Figure 10, where the stabilizing role of $\beta$ is evident. For $\beta=0$ there is no fraction evolution and we always have 50 rational firms out of 100 , but the dynamics of production levels are chaotic. As $\beta$ increases, the dynamics of quantities and fractions become qualitatively 


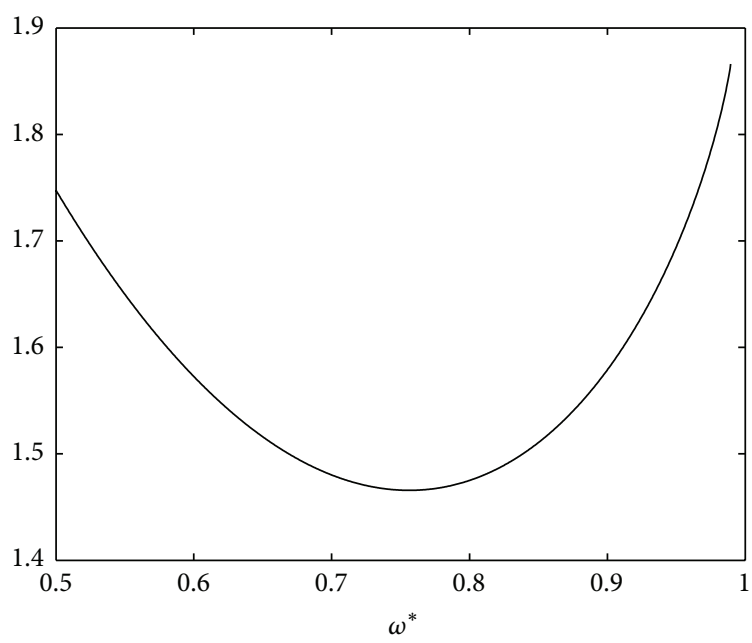

Figure 8: Plot of the 1.h.s. of (64) with respect to the possible equilibrium fractions $\omega^{*} \in[1 / 2,1-1 / N)$.

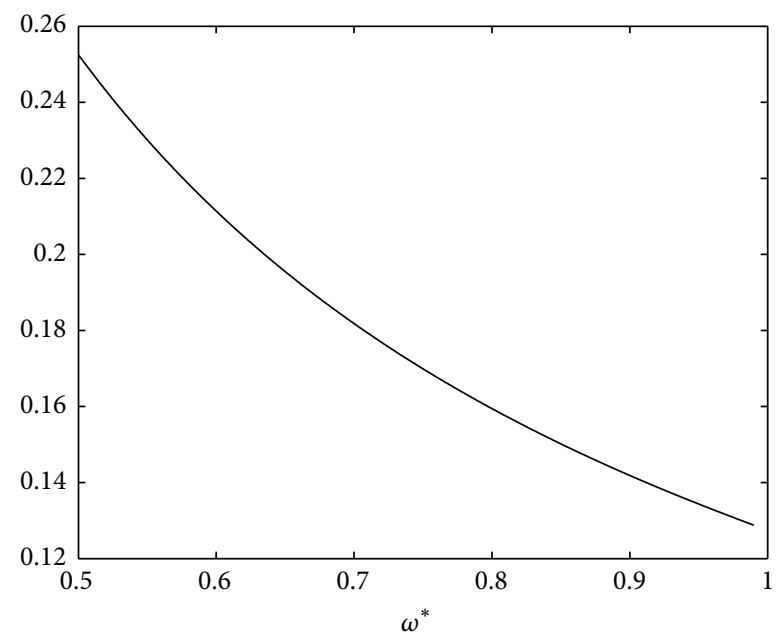

Figure 9: Plot of the 1.h.s. of (65) with respect to the possible equilibrium fractions $\omega^{*} \in[1 / 2,1-1 / N)$.

more stable, and they converge to the equilibrium for $\beta>$ 0.96 . In particular, for $\beta=0.877$ we have that the equilibrium fraction is $\omega^{*}=0.78$ and the equilibrium strategy is $q_{2}^{*}=$ 9.265 , for which the eigenvalues of $J_{G}\left(q_{2}^{*}, \omega^{*}\right)$ are now $\lambda_{1}=$ -1.032 and $\lambda_{2}=0.123$, which gives an unstable dynamic. Conversely, for $\beta=0.962$ we have that the equilibrium fraction is $\omega^{*}=0.8$ and the equilibrium strategy is $q_{2}^{*}=$ 9.249 , for which the eigenvalues of $J_{G}\left(q_{2}^{*}, \omega^{*}\right)$ are now $\lambda_{1}=$ -0.99 and $\lambda_{2}=0.127$, which means that the equilibrium is stable. It is interesting to look also at the profit differential evolution, which for small values of $\beta$ oscillates between positive and negative values, while for larger values it is always negative.

This allows us to say that, referring to particular parameter sets, different behaviors with respect to $\beta$ are possible. In particular, the neutral and stabilizing roles of $\beta$ are very interesting as in the existing literature evolutionary pressure usually introduces instability. The discriminating factor is for us the net profit differential. When the marginal costs are identical, as in the previous section and in [30], the equilibrium profits are the same and the net profit is favorable to the best response firms, due to the informational costs of the rational ones. Hence, rational firms are inclined to switch to the best response mechanism and this shifts the oligopoly composition towards unstable configurations, especially when the evolutionary pressure is sufficiently large. Profits of the rational firms are smaller than those of the best response firms also when $c_{1}<c_{2}$ but the difference is sufficiently small and when $c_{1}>c_{2}$. Conversely, when $c_{1}<c_{2}$ and the difference is sufficiently large, we have $\pi_{1}\left(q_{2}^{*}, \omega^{*}\right)-$ $C>\pi_{2}\left(q_{2}^{*}, \omega^{*}\right)$, so that the best response firms switch to the more profitable rational mechanism, and this can both introduce or not affect stability.

\section{Conclusions}

The general setting we considered allowed us to show that the oligopoly size, the number of boundedly rational players, and the evolutionary pressure do not always have a destabilizing role. Even if the economic setting and the considered behavioral rules are the same as in [30], the model we dealt with gave us the possibility to investigate a wide range of scenarios which are not present in [30]. Indeed, the explicit introduction of the oligopoly size in the model allowed us to analyze in an heterogeneous context the effects of the firms number on the stability of the equilibrium. We found a general confirmation of a behavior which is well known in the homogeneous oligopoly framework; that is, increasing the firms number $N$ may lead the equilibrium to instability $($ see $[2,3]$ for the linear context and, for nonlinear settings, $[12,45,46]$ where isoelastic demand functions are taken into account). However, we showed that the presence of both rational and naive firms can give rise to oligopoly compositions which are stable for any oligopoly size, if the fraction of "stabilizing" rational firms is sufficiently large. Similarly, we showed that even if in general replacing rational firms with best response firms (i.e., decreasing $\omega$ ) introduces instability, for particular parameter configurations all the (heterogeneous) oligopoly compositions of a fixed size are stable. Hence, in the linear context analyzed in the present paper the role of $\omega$ is (nearly) intuitive, that is, increasing the number of rational agents stabilizes the system or varying $\omega$ does not alter the stability. On the other hand, in nonlinear contexts this may not be true anymore. For instance, in the working paper [18], where we deal with an isoelastic demand function, we find that increasing the number of rational firms may destabilize the system, under suitable conditions on marginal costs. It would be also interesting to analyze nonlinear frameworks with multiple Nash equilibria, in order to investigate the role of $\omega$ in such a different context, as well.

The results recalled above are obtained in the present paper for the fixed fraction framework, in which we proved that stability is not affected by cost differences among the firms. Conversely, regarding the evolutionary fraction setting we showed that cost differences may be relevant for stability. In fact, even if for identical marginal costs we analytically 

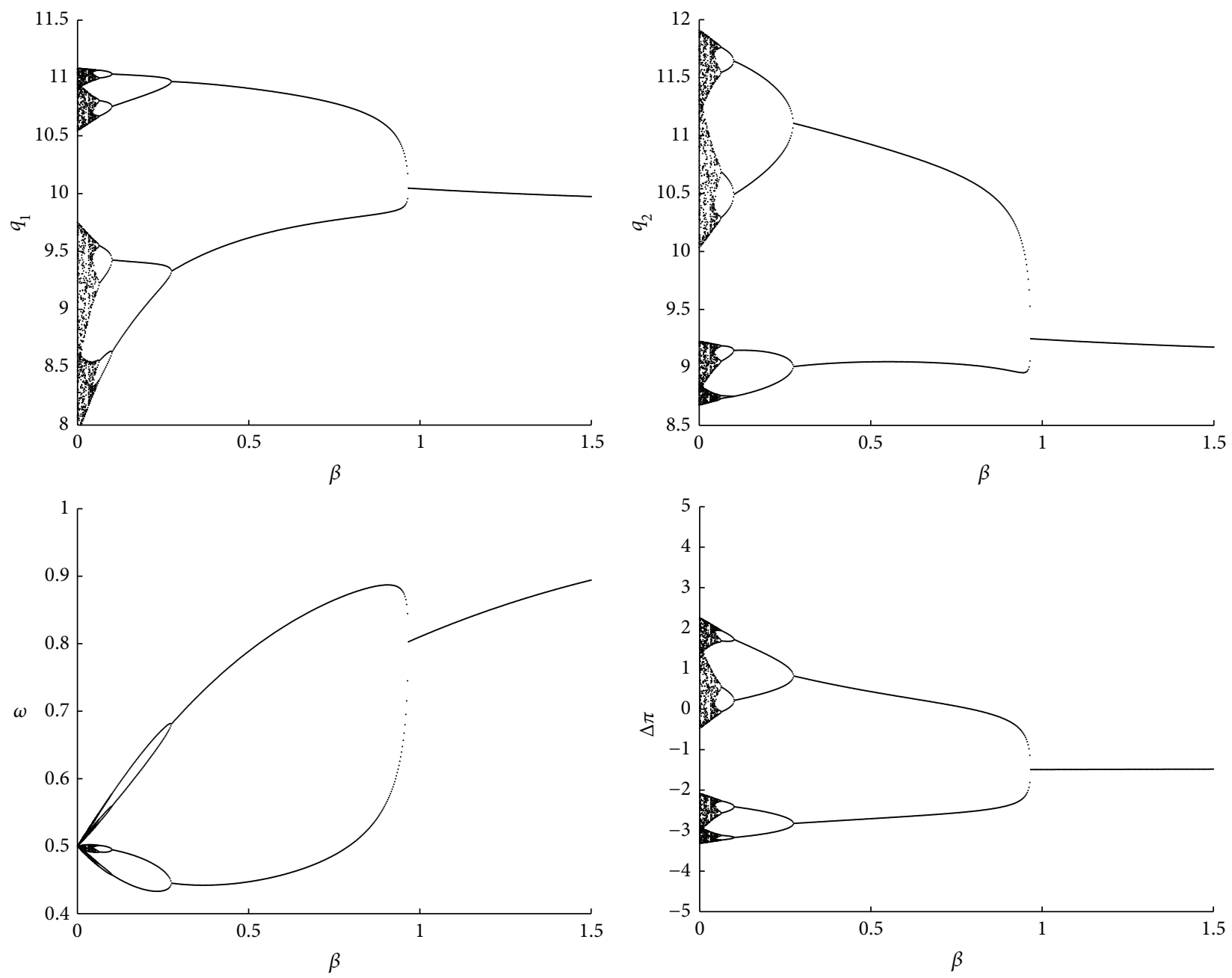

FIGURE 10: Bifurcation diagrams, related to the stabilizing scenario for $\beta$, of quantities $q_{1}$ and $q_{2}$, of fraction $\omega$ and of the net profit differential $\Delta \pi$. The initial composition corresponding to $\beta=0$ is unstable but, since the marginal costs are sufficiently favorable to the rational firms, increasing the propensity to switch stabilizes the dynamics.

proved the destabilizing role of the evolutionary pressure and informational costs, in agreement with the results in [30], we showed that the stability can be improved or at least preserved by the intensity of switching if rational firms are efficient enough with respect to the best response firms. We have been able to prove this behavior only for particular parameter choices, due to the high complexity of the analytic computations. This last scenario definitely requires further investigations, in order to extend the results to a wider range of parameters and to test what happens in different economic settings, considering, for example, a nonlinear demand function. Other generalizations may concern the involved decisional rules, taking into account agents with further reduced rationality, heuristics which are not based on a best response mechanism, or even more complex scenarios, where more than two behavioral rules are considered or where the firms adopting the same behavioral rules are not identical, also in view of facing empirical and practical market contexts.

\section{Conflict of Interests}

The authors declare that there is no conflict of interests regarding the publication of this paper.

\section{Acknowledgments}

The authors thank the anonymous reviewer for the helpful and valuable comments.

\section{References}

[1] A. A. Cournot, Reserches sur les Principles Mathematiques de la Theorie des Richesses, Hachette, Paris, France, 1838.

[2] T. F. Palander, "Konkurrens och marknadsjamvikt vid duopol och oligopol," Ekonomisk Tidskrift, vol. 41, pp. 124-250, 1939.

[3] R. D. Theocharis, "On the stability of the Cournot solution on the oligopoly problem," The Review of Economic Studies, vol. 27, no. 2, pp. 133-134, 1960. 
[4] M. Lampart, "Stability of the Cournot equilibrium for a Cournot oligopoly model with $n$ competitors," Chaos, Solitons \& Fractals, vol. 45, no. 9-10, pp. 1081-1085, 2012.

[5] A. Matsumoto and F. Szidarovszky, "Stability, bifurcation, and chaos in N-firm nonlinear Cournot games," Discrete Dynamics in Nature and Society, vol. 2011, Article ID 380530, 22 pages, 2011.

[6] A. K. Naimzada and F. Tramontana, "Controlling chaos through local knowledge," Chaos, Solitons \& Fractals, vol. 42, no. 4, pp. 2439-2449, 2009.

[7] G. I. Bischi, C. Chiarella, M. Kopel, and F. Szidarovszky, Nonlinear Oligopolies: Stability and Bifurcations, Springer, Berlin, Germany, 2009.

[8] H. N. Agiza and A. A. Elsadany, "Nonlinear dynamics in the Cournot duopoly game with heterogeneous players," Physica A: Statistical Mechanics and its Applications, vol. 320, no. 1-4, pp. 512-524, 2003.

[9] H. N. Agiza and A. A. Elsadany, "Chaotic dynamics in nonlinear duopoly game with heterogeneous players," Applied Mathematics and Computation, vol. 149, no. 3, pp. 843-860, 2004.

[10] H. N. Agiza, A. S. Hegazi, and A. A. Elsadany, "Complex dynamics and synchronization of a duopoly game with bounded rationality," Mathematics and Computers in Simulation, vol. 58, no. 2, pp. 133-146, 2002.

[11] H. N. Agiza, E. M. Elabbasy, and A. A. Elsadany, "Complex dynamics and chaos control of heterogeneous quadropoly game," Applied Mathematics and Computation, vol. 219, no. 24, pp. 11110-11118, 2013.

[12] E. Ahmed and H. N. Agiza, "Dynamics of a Cournot game with n-competitors," Chaos, Solitons \& Fractals, vol. 9, no. 9, pp. 15131517, 1998.

[13] N. Angelini, R. Dieci, and F. Nardini, "Bifurcation analysis of a dynamic duopoly model with heterogeneous costs and behavioural rules," Mathematics and Computers in Simulation, vol. 79, no. 10, pp. 3179-3196, 2009.

[14] G. I. Bischi and A. Naimzada, "Global analysis of a dynamic duopoly game with bounded rationality," in Advances in Dynamic Games and Applications, J. Filar, V. Gaitsgory, and K. Mizukami, Eds., vol. 5 of Annals of the International Society of Dynamic Games, pp. 361-385, Birkhäuser, Boston, Mass, USA, 2000.

[15] J. S. Canovas, T. Puu, and M. Ruiz, "The Cournot-Theocharis problem reconsidered," Chaos, Solitons \& Fractals, vol. 37, no. 4, pp. 1025-1039, 2008.

[16] F. Cavalli and A. Naimzada, "A Cournot duopoly game with heterogeneous players: nonlinear dynamics of the gradient rule versus local monopolistic approach," Applied Mathematics and Computation, vol. 249, pp. 382-388, 2014.

[17] F. Cavalli and A. Naimzada, "Nonlinear dynamics and convergence speed of heterogeneous Cournot duopolies involving best response mechanisms with different degrees of rationality," Nonlinear Dynamics, 2015.

[18] F. Cavalli, A. Naimzada, and M. Pireddu, "Heterogeneity and the (de)stabilizing role of rationality," To appear in Chaos, Solitons \& Fractals.

[19] F. Cavalli, A. Naimzada, and F. Tramontana, "Nonlinear dynamics and global analysis of a heterogeneous Cournot duopoly with a local monopolistic approach versus a gradient rule with endogenous reactivity," Communications in Nonlinear Science and Numerical Simulation, vol. 23, no. 1-3, pp. 245-262, 2015.
[20] E. M. Elabbasy, H. N. Agiza, and A. A. Elsadany, "Analysis of nonlinear triopoly game with heterogeneous players," Computers \& Mathematics with Applications, vol. 57, no. 3, pp. 488-499, 2009.

[21] W. Ji, "Chaos and control of game model based on heterogeneous expectations in electric power triopoly," Discrete Dynamics in Nature and Society, vol. 2009, Article ID 469564, 8 pages, 2009.

[22] T. Li and J. Ma, "The complex dynamics of R\&D competition models of three oligarchs with heterogeneous players," Nonlinear Dynamics, vol. 74, no. 1-2, pp. 45-54, 2013.

[23] A. Matsumoto, "Statistical dynamics in piecewise linear Cournot game with heterogeneous duopolists," International Game Theory Review, vol. 6, no. 3, pp. 295-321, 2004.

[24] A. K. Naimzada and L. Sbragia, "Oligopoly games with nonlinear demand and cost functions: two boundedly rational adjustment processes," Chaos, Solitons \& Fractals, vol. 29, no. 3, pp. 707-722, 2006.

[25] X. Pu and J. Ma, "Complex dynamics and chaos control in nonlinear four-oligopolist game with different expectations," International Journal of Bifurcation and Chaos in Applied Sciences and Engineering, vol. 23, no. 3, Article ID 1350053, 15 pages, 2013.

[26] F. Tramontana, "Heterogeneous duopoly with isoelastic demand function," Economic Modelling, vol. 27, no. 1, pp. 350-357, 2010.

[27] J. Tuinstra, "A price adjustment process in a model of monopolistic competition," International Game Theory Review, vol. 6, no. 3, pp. 417-442, 2004.

[28] M. Anufriev, D. Kopányi, and J. Tuinstra, "Learning cycles in Bertrand competition with differentiated commodities and competing learning rules," Journal of Economic Dynamics and Control, vol. 37, no. 12, pp. 2562-2581, 2013.

[29] G. I. Bischi, F. Lamantia, and D. Radi, "An evolutionary Cournot model with limited market knowledge," To appear in Journal of Economic Behavior \& Organization.

[30] E. Droste, C. Hommes, and J. Tuinstra, "Endogenous fluctuations under evolutionary pressure in Cournot competition," Games and Economic Behavior, vol. 40, no. 2, pp. 232-269, 2002.

[31] J.-G. Du, Y.-Q. Fan, Z.-H. Sheng, and Y.-Z. Hou, "Dynamics analysis and chaos control of a duopoly game with heterogeneous players and output limiter," Economic Modelling, vol. 33, pp. 507-516, 2013.

[32] A. Naimzada and M. Pireddu, "Dynamics in a nonlinear Keynesian good market model," Chaos, vol. 24, no. 1, Article ID 013142, 2014.

[33] A. Naimzada and M. Pireddu, "Dynamic behavior of product and stock markets with a varying degree of interaction," Economic Modelling, vol. 41, pp. 191-197, 2014.

[34] W. A. Brock and C. H. Hommes, "A rational route to randomness," Econometrica, vol. 65, no. 5, pp. 1059-1095, 1997.

[35] A. Banerjee and J. W. Weibull, "Neutrally stable outcomes in cheap-talk coordination games," Games and Economic Behavior, vol. 32, no. 1, pp. 1-24, 2000.

[36] D. Gale and R. W. Rosenthal, "Experimentation, imitation, and stochastic stability," Journal of Economic Theory, vol. 84, no. 1, pp. 1-40, 1999.

[37] X.-Z. He and F. H. Westerhoff, "Commodity markets, price limiters and speculative price dynamics," Journal of Economic Dynamics and Control, vol. 29, no. 9, pp. 1577-1596, 2005. 
[38] Z. Sheng, J. Du, Q. Mei, and T. Huang, "New analyses of duopoly game with output lower limiters," Abstract and Applied Analysis, vol. 2013, Article ID 406743, 10 pages, 2013.

[39] C. Wagner and R. Stoop, "Optimized chaos control with simple limiters," Physical Review E-Statistical, Nonlinear, and Soft Matter Physics, vol. 63, Article ID 017201, 2001.

[40] C. Wieland and F. H. Westerhoff, "Exchange rate dynamics, central bank interventions and chaos control methods," Journal of Economic Behavior \& Organization, vol. 58, no. 1, pp. 117-132, 2005.

[41] G. I. Bischi, R. Dieci, G. Rodano, and E. Saltari, "Multiple attractors and global bifurcations in a Kaldor-type business cycle model," Journal of Evolutionary Economics, vol. 11, no. 5, pp. 527-554, 2001.

[42] J. K. Goeree, C. Hommes, and C. Weddepohl, "Stability and complex dynamics in a discrete tâtonnement model," Journal of Economic Behavior \& Organization, vol. 33, no. 3-4, pp. 395-410, 1998.

[43] J. K. Hale and H. Koçak, Dynamics and Bifurcations, vol. 3 of Texts in Applied Mathematics, Springer, New York, NY, USA, 1991.

[44] E. I. Jury, Theory and Application of the z-Transform Method, John Wiley \& Sons, New York, NY, USA, 1964.

[45] H. N. Agiza, "Explicit stability zones for Cournot games with 3 and 4 competitors," Chaos, Solitons \& Fractals, vol. 9, no. 12, pp. 1955-1966, 1998.

[46] T. Puu, "On the stability of Cournot equilibrium when the number of competitors increases," Journal of Economic Behavior and Organization, vol. 66, no. 3-4, pp. 445-456, 2008. 


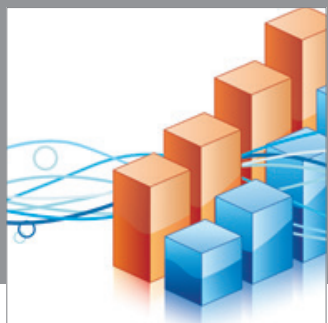

Advances in

Operations Research

mansans

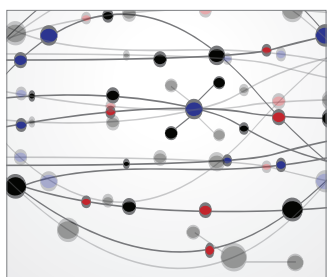

The Scientific World Journal
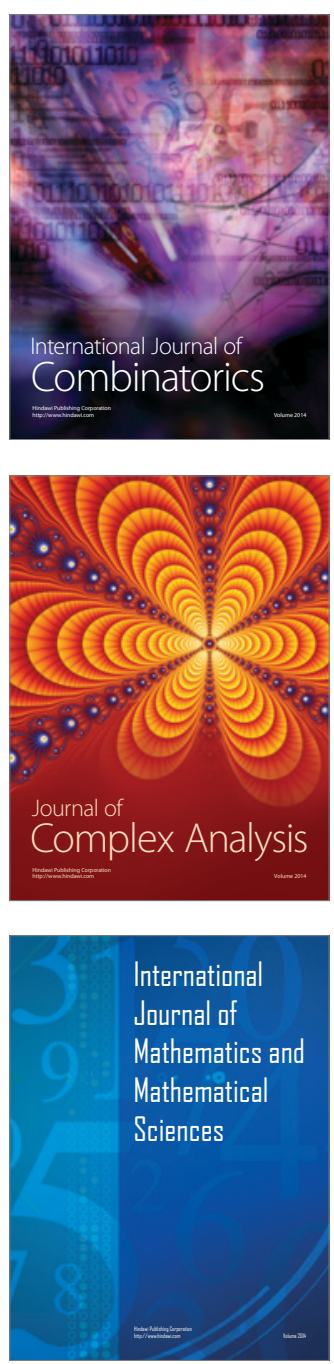
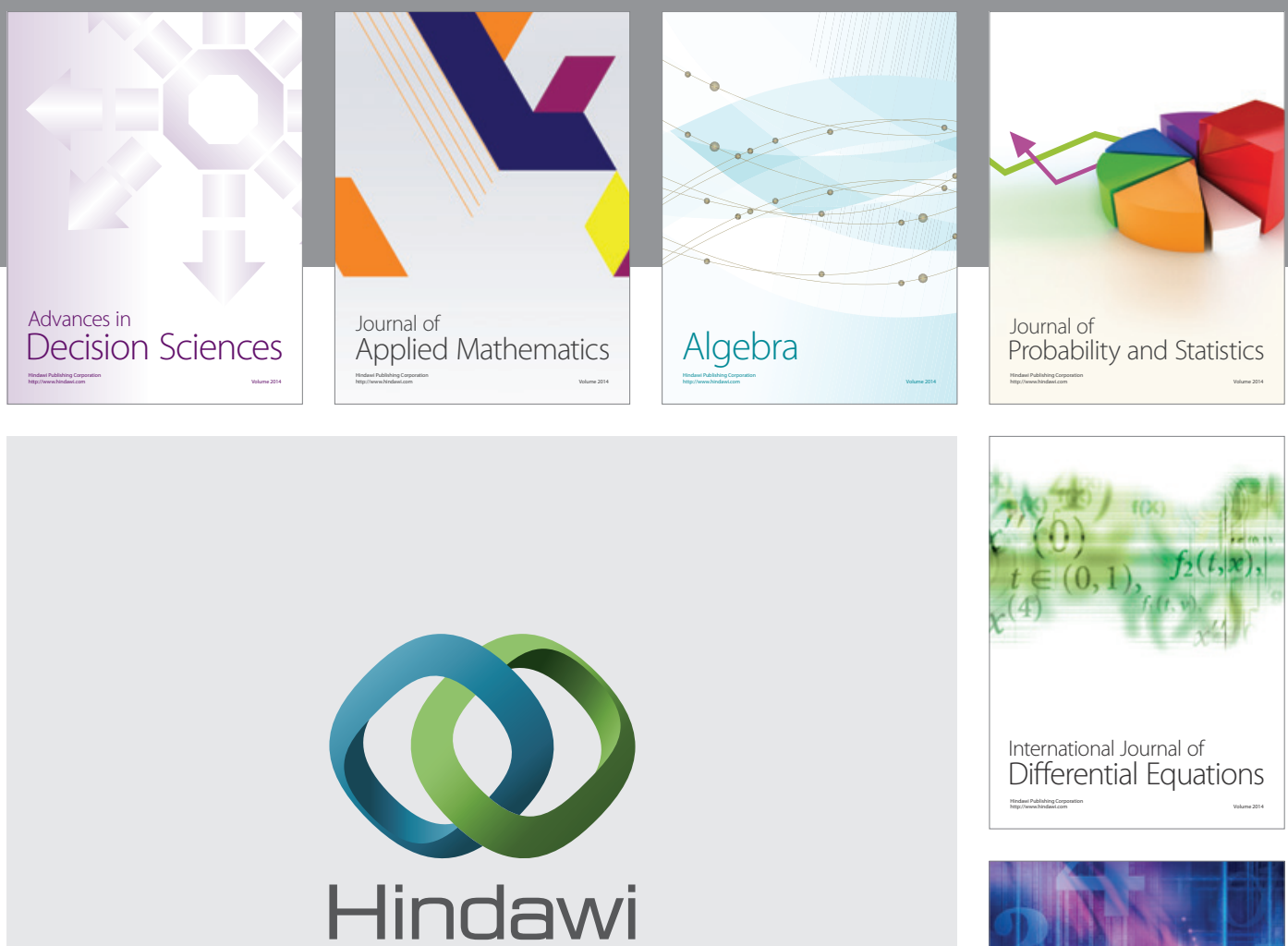

Submit your manuscripts at http://www.hindawi.com
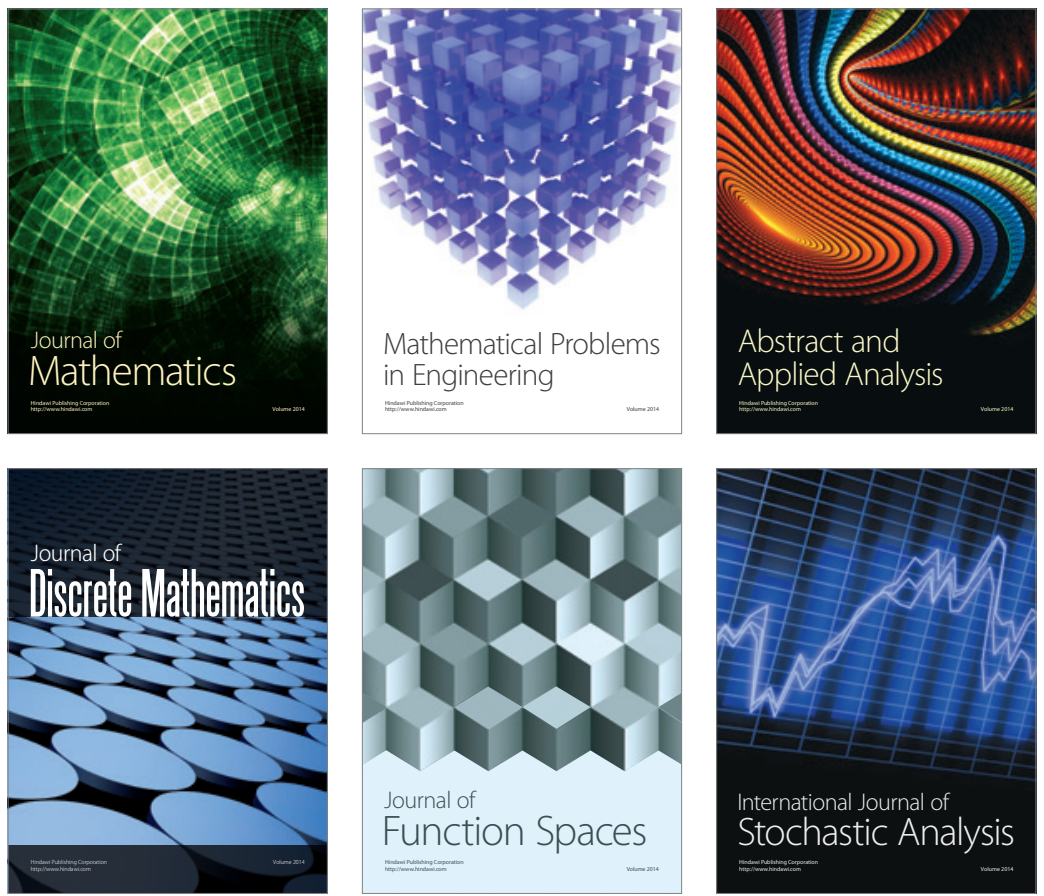

Journal of

Function Spaces

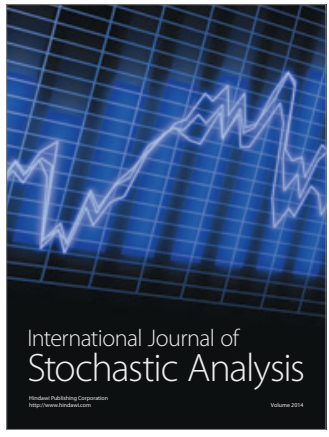

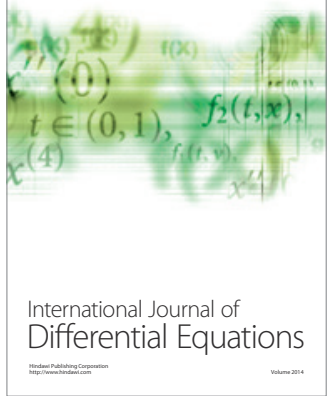
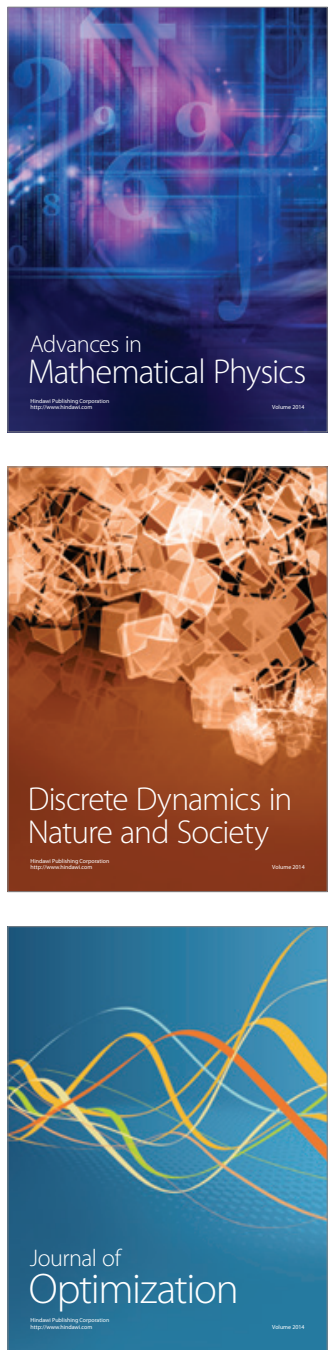